\title{
Deep inelastic scattering on an extremal Reissner-Nordström-AdS black hole. II. Holographic fermi surface
}

\author{
Kiminad A. Mamo* and Ismail Zahed \\ Department of Physics and Astronomy, Stony Brook University, Stony Brook, New York 11794-3800, USA
}

(Received 4 June 2019; accepted 27 February 2020; published 17 March 2020)

\begin{abstract}
We consider deep inelastic scattering (DIS) on a dense nucleus described as an extremal RN-AdS black hole with holographic quantum fermions in the bulk. We evaluate the 1-loop fermion contribution to the R-current on the charged black hole, and map it on scattering off a Fermi surface of a dense and large nucleus with fixed atomic number. Near the black hole horizon, the geometry is that of $\mathrm{AdS}_{2} \times \mathrm{R}^{3}$ where the fermions develop an emergent Fermi surface with anomalous dimensions. DIS scattering off these fermions yields to anomalous partonic distributions mostly at large-x, as well as modified hard scattering rules. The pertinent R-ratio for the black hole is discussed. For comparison, the structure functions and the R-ratio in the probe or dilute limit with no backreaction on the geometry, are also derived. We formulate a hybrid holographic model for DIS scattering on heavy and light nuclei, which compares favorably to the existing data for $\mathrm{Pb}, \mathrm{Au}, \mathrm{Fe}, \mathrm{C}$, and $\mathrm{He}$ over a wide range of parton-X.
\end{abstract}

DOI: 10.1103/PhysRevD.101.066014

\section{INTRODUCTION}

Many years ago the EMC collaboration at CERN has revealed that deep inelastic scattering (DIS) on an iron nucleus deviates substantially from deuterium [1] contrary to established lore. Since then, many other collaborations using both electron and muon probes have confirmed this observation [2-4]. Although the nucleus is a collection of loosely bound nucleons with confined quarks, DIS scattering is much richer in a nucleus. The nuclear structure functions display shadowing at low-x, a depletion at intermediate- $x$, and an enhancement due mostly to Fermi motion at large-x.

QCD supports the idea that hadrons are composed of quarks and gluons as revealed by DIS scattering of electrons on nucleons at SLAC. The scaling laws initially reported follows from scattering on pointlike object or partons. Because of asymptotic freedom, the partons interact weakly at short distances leading to relatively small scaling violations at intermediate-x. At low-x, perturbative QCD predicts a large enhancement in the nucleon structure functions due to the rapid growth of the gluons [5] that eventually saturate [6]. This observation has been confirmed at HERA [7,8].

\footnotetext{
*kiminad.mamo@stonybrook.edu

ismail.zahed@stonybrook.edu
}

Published by the American Physical Society under the terms of the Creative Commons Attribution 4.0 International license. Further distribution of this work must maintain attribution to the author(s) and the published article's title, journal citation, and DOI. Funded by SCOAP ${ }^{3}$.
DIS in holography at moderate-x is different from weak coupling as it involves hadronic and not partonic constituents [9]. The large gauge coupling causes the charges to rapidly deplete their energy and momentum, making them invisible to hard probes. However, because the holographic limit enjoys approximate conformal symmetry, the form factors exhibit various scaling laws including the partoncounting rules [10]. The holographic structure functions fail to reproduce the Callan-Gross sum rule [9] at intermediate- $x$, but agree with it at large- $x$ when the parton momentum fraction is in the neighborhood of 1 [11]. In contrast, DIS scattering at low-x on a non-extremal thermal black hole was argued to be partonic and fully saturated [12].

This paper is a follow up on our recent investigation of DIS scattering on a nucleus as an extremal RN-AdS black hole [13]. In the double limit of a large number of colors and gauge coupling, the leading contribution amounts to the Abelian part of the R-current being absorbed in bulk by the black hole. After mapping at the boundary, the ensuing nuclear structure functions show strong shadowing at low-x, but wane exponentially for large- $x$ as originally noted for the thermal black hole in [12].

At next to leading-order, the R-current scatters off charged fermionic pairs forming a holographic Fermi liquid around the black hole. The purpose of this paper is to detail DIS scattering on this dense holographic liquid as the analogue of DIS scattering on a nucleus described as a Fermi liquid. Some aspects of this liquid near the horizon were initially discussed in lower dimensions [14]. It should be noted that at next to leading order the R-current scatters also off bulk charged scalars. However, these scalars are 
bosonic and do not form a Fermi surface. Since the main thrust of the paper is to probe the role of the quantum corrections induced by a bulk Fermi surface to the leading black-hole contribution as for a realistic DIS process on a nucleus, the contribution of the quantum scalar corrections to the DIS process will not be considered in this work.

Standard DIS scattering on a nucleus is mostly on a Fermi gas in a mean field "trap," so the present calculations show how the same scattering operates on an emergent Fermi surface with strongly coupled fermionic constituents in a trap produced by a charged black hole. A chief observation is that the partonic structure functions at large- $x$, are modified by an emergent Fermi surface. The latter follows from an $\mathrm{AdS}_{2} \times \mathrm{R}^{3}$ reduction of the geometry near the black-hole horizon, and asymptotes a warped Fermi liquid near the boundary. The corresponding R-ratio exhibits shadowing at very low- $\mathrm{x}$, antishadowing at intermediate- $x$ and Fermi motion at large-x, much like the $\mathrm{R}$-ratio for DIS scattering on finite nuclei. Shadowing is caused by the coherent many-body effects and is captured by DIS scattering in leading order on the black hole at low-x, while Fermi motion at large- $x$ is due to the incoherent scattering on quantum fermions around the black hole in the form of a holographic Fermi surface.

This paper consists of several new results: (1) an explicit derivation of the structure functions for DIS scattering on the emerging holographic Fermi surface around an extremal black hole; (2) the characterization of these structure functions both at large-x and low-x, with the identification of new anomalous exponents at large $\mathrm{x}$; (3) an explicit derivation of the R-ratio for DIS scattering on the extremal black hole as a model for DIS scattering on a dense and finite nucleus; (4) an explicit derivation of the same structure functions in the probe fermion limit as a model for DIS scattering on a dilute nucleus; (5) a comparative study of the R-ratio in the probe limit; (6) a detailed comparison to the empirical DIS scattering data from light to heavy nuclei.

The organization of the paper is as follows: in Sec. II, we briefly review the setting for the extremal RN-AdS black hole, and the key characteristics of the holographic Fermi liquid. In Sec. III, we derive the contribution to the boundary effective action of an R-photon scattering off bulk quantum fermions. The result is quantum and dominant at large- $\mathrm{x}$, and corrects the classical and leading contribution from the bulk black hole. In Sec. IV, we analyze the contribution stemming from the quantum fermions near the horizon. In Sec. V, we detail our derivation of the R-ratio for DIS scattering on a dense nucleus as quantum corrected holographic black hole. For comparison, we discuss in Sec. VI the probe or dilute limit with the bulk fermions carrying a finite density in AdS without affecting the underlying geometry. The pertinent $\mathrm{R}$-ratio in this regime is derived and analyzed. In Sec. VII we motivate a hybrid holographic model for DIS scattering (a)

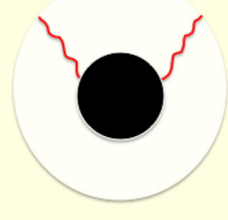

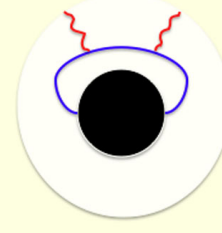

(b)
FIG. 1. Absorptive part of the R-current on a nucleus as an extremal RN-AdS black hole: (a) absorptive contribution to order $N_{c}^{2}$; (b) absorptive fermionic contribution to order $N_{c}^{0}$.

on light and heavy nuclei which compares favorably to the existing world-data for a wide range of parton-x. Our conclusions are in Sec. VIII. Some useful details are found in several Appendixes.

\section{EXTREMAL BLACK HOLE: DENSE LIMIT}

In this work we will address DIS scattering on a cold and dense nucleus as a dual to an RN-AdS black hole following on our recent analysis [15]. Conventional DIS scattering on cold nuclei with many of the conventions used are reviewed in [16]. In holography, DIS scattering on a nucleus as an RN-AdS black hole is illustrated in Fig. 1. In the holographic limit, the leading contribution is Fig. 1(a) with the structure functions being the absorbed parts of the R-current. To this order, the structure functions have considerable support mostly at low-x [13] (see below). At next-to-leading order, the R-current is absorbed through the virtual fermionic loop shown in Fig. 1(b). This loop describes a fermionic hallow around the RN-AdS black hole that acts as a holographic Fermi liquid. Below we detail how this contribution leads to structure functions with mostly support at large-x. This description is complementary to our recent analysis based on a generic density expansion around a trapped Fermi liquid [15].

\section{A. The extremal and charged black hole}

The RN-AdS black hole is described by effective gravity coupled to a U(1) gauge field in a 5-dimensional curved AdS space [17]

$S=\frac{1}{2 \kappa^{2}} \int d^{5} x \sqrt{-g}(\mathbb{R}-2 \Lambda)-\frac{1}{4 e^{2}} \int d^{5} x \sqrt{-g} F^{2}$.

The Ricci scalar is $\mathbb{R}$, and $\kappa^{2}=8 \pi G_{5}$ and $\Lambda=-6 / R^{2}$ are the gravitational and cosmological constant. The curvature radius of the AdS space is $R$ with line element

$$
d s^{2}=\frac{r^{2}}{R^{2}}\left(-f d t^{2}+d \vec{x}^{2}\right)+\frac{R^{2}}{r^{2} f} d r^{2}
$$

and warping factor 


$$
f(r)=\left(1-\frac{r_{+}^{2}}{r^{2}}\right)\left(1-\frac{r_{-}^{2}}{r^{2}}\right)\left(1+\frac{r_{+}^{2}}{r^{2}}+\frac{r_{-}^{2}}{r^{2}}\right)
$$

with $\quad r_{+}>r_{-}$the outer-inner horizons satisfying $f\left(r_{ \pm}\right)=0$.

The black hole is charged and sources the R-potential

$$
A_{t}=\mu-\frac{Q}{r^{2}}
$$

provided that the electric charge $Q$ and the geometrical charge $q$ satisfy

$$
\frac{q^{2} R^{2}}{Q^{2}}=\frac{4}{3} \times \frac{2 \kappa^{2}}{4 e^{2}}=\frac{R^{2}}{6 \tilde{\alpha}}
$$

where

$$
\begin{aligned}
2 \kappa^{2} & =\frac{8 \pi^{2} R^{3}}{N_{c}^{2}} \\
4 e^{2} & =\tilde{\alpha} \frac{64 \pi^{2} R}{N_{c}^{2}} .
\end{aligned}
$$

We have defined $\tilde{\alpha}=1$ for a U(1) R-charge, and $\tilde{\alpha}=\frac{1}{4} \frac{N_{c}}{N_{f}}$ for a D3-D7 U(1) vector charge. The temperature of the $\mathrm{RN}$-AdS black hole is

$$
T=\frac{r_{+}^{2} f^{\prime}\left(r_{+}\right)}{4 \pi R^{2}}=\frac{r_{+}}{\pi R^{2}}\left(1-\frac{\mu^{2} \pi^{2} R^{4} \gamma^{2}}{r_{+}^{2}}\right)
$$

with $\gamma^{2}=1 / 12 \pi^{2} \tilde{\alpha}$. The chemical potential $\mu$ is fixed by the zero potential condition on the outer horizon $A_{t}\left(r_{+}\right)=0$ or $\mu=Q / r_{+}^{2}$. At extremality where $T=0$, we have $r_{+}=r_{-}=\pi R^{2} \gamma \mu=\frac{R^{2}}{2 \sqrt{3}} \sqrt{\tilde{\alpha}}$.

\section{B. Holographic Fermi liquid}

The fermionic fields in bulk are characterized by the Dirac action in a charged AdS black hole geometry

$$
S=-\int d^{5} x \sqrt{-g} i\left(\bar{\psi} \Gamma^{M} \mathcal{D}_{M} \psi-m \bar{\psi} \psi\right)
$$

with $\bar{\psi}=\psi^{\dagger} \Gamma^{\underline{t}}$, and the long derivative

$$
\mathcal{D}_{M}=\partial_{M}+\frac{1}{4} \omega_{a b M} \Gamma^{a b}-i e_{R} A_{M}
$$

The indices $M, N \cdots$ or $\mu, \nu, r \cdots$ refer to the space-time indices, and $a, b, \cdots$ to space-time indices with underline correspond to tangent space indices. Therefore, for example, $\Gamma^{a}$ denotes the gamma matrices in the tangent space, $\Gamma^{M}$ denotes gamma matrices in the curved spacetime. They are specifically given in Appendix A.
A bulk fermion field of mass $m$ and R-charge $e_{R}$ is dual to a composite boundary field of conformal dimension $\Delta=\frac{3}{2}+m R$. Since the horizon of the extremal charged $\mathrm{RN}$-AdS black is characterized by a finite U(1) electric field, fermionic pair creation takes place through the Schwinger mechanism. As a result, the black hole say with positive R-charge absorbs the negative part of the pairs and expel the positive part. Since AdS is hyperbolic and confining, the positive charge falls back to the surface of the black hole, accumulating into a hallow or holographic Fermi liquid.

The characteristics of the low-lying excitations of the holographic Fermi liquid for low frequencies $\left|k^{0}\right|<\mu$ and low momenta $k=|\vec{k}|$, have been discussed in $[18,19]$. In particular, near the horizon the $\mathrm{AdS}_{5}$ geometry factors into $\mathrm{AdS}_{2} \times \mathrm{R}^{3}$.

\section{Case-1: $e_{R}^{2} \tilde{\alpha}<\frac{1}{4}(m R)^{2}$}

The fermions exhibit strong distortion in the $\mathrm{AdS}_{2}$ geometry, with [18]

$$
\mathcal{G}_{R}^{11}\left(k^{0}, \vec{k}\right)=C(\vec{k})\left(k^{0}\right)^{2 \nu_{k}}\left(\begin{array}{cc}
0 & 0 \\
0 & 1
\end{array}\right),
$$

and

$$
\begin{aligned}
\nu_{k} & =\frac{\sqrt{\tilde{\alpha}}}{\mu}\left(k^{2}-k_{R}^{2}\right)^{\frac{1}{2}} \\
& \equiv \frac{\sqrt{\tilde{\alpha}}}{\mu}\left(k^{2}-\frac{\mu^{2}}{3 \tilde{\alpha}}\left(e_{R}^{2} \tilde{\alpha}-\frac{1}{4}(m R)^{2}\right)\right)^{\frac{1}{2}} .
\end{aligned}
$$

Note that $k_{R}^{2}<0$ in this case, i.e., for $e_{R}^{2} \tilde{\alpha}<\frac{1}{2}(m R)^{2}$.

Throughout, we will use the block notation to refer to the fermionic retarded (Feynman) propagators

$$
\mathcal{G}_{R, F}=\left(\begin{array}{ll}
\mathcal{G}^{11} & \mathcal{G}^{12} \\
\mathcal{G}^{21} & \mathcal{G}^{22}
\end{array}\right)_{R, F}
$$

\section{Case-2: $e_{R}^{2} \tilde{\alpha}>\frac{1}{4}(m R)^{2}$}

For $k_{R}^{2}>0$ and $k \leq k_{R}$, the corresponding holographic spectral function exhibits oscillating behavior and gapless excitations, with comparable real and imaginary parts. In other words, the excitations in this oscillating region are short lived as they form and quickly fall into the extremal RN-AdS black hole.

Further arguments [18,19] show that the fermionic density diverges near the horizon causing strong back reaction. As a result, the near horizon geometry becomes a Lifshitz geometry whereby the Fermi-like volume is resolved into concentric Fermi spheres each describing heavy Fermions with narrow widths, thereby explaining the gapless like excitations. This resolution occurs only for $\left|k^{0}\right| / \mu \sim e^{-N_{c}^{2}}$ and resorbs for $\left|k^{0}\right| / \mu \sim N_{c}^{0}$. 


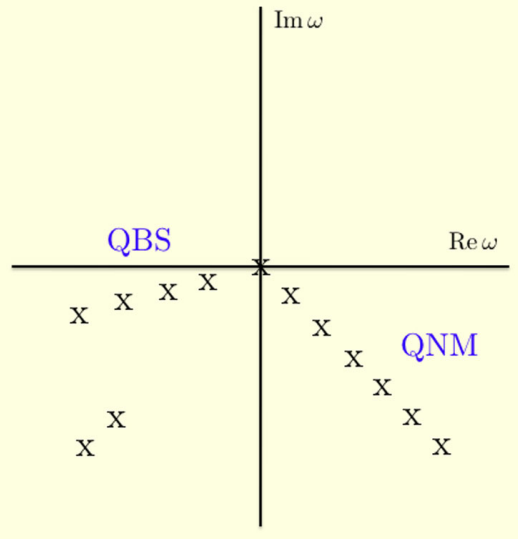

FIG. 2. Schematic description of the poles of the Green function. For sufficiently large U(1) charge $e_{R}$ some of the quasinormal modes (QNM) of the RN-AdS black hole transmute to narrow quasibound states (QBS) by moving closer to the real-axis.

\section{Case-3: $e_{R}^{2} \tilde{\alpha}>\frac{1}{4}(m R)^{2}$}

For $k_{R}^{2}>0$ and $k \geq k_{R}$, localized and long lived fermionic states emerge that are characterized by a Fermi momentum $k_{F}>k_{R}$. In this case, the retarded propagator near the Fermi surface reads $[18,19]$

$$
\mathcal{G}_{R}^{11}\left(k^{0}, \vec{k}\right) \approx \frac{h_{1}}{k-k_{F}-\frac{1}{v_{F}} k^{0}-\Pi\left(k^{0}\right)}\left(\begin{array}{cc}
0 & 0 \\
0 & 1
\end{array}\right)
$$

with

$$
\begin{aligned}
\Pi\left(k^{0}\right) & =h_{2} e^{i \gamma_{k_{F}}}\left(k^{0}\right)^{2 \nu_{k}} \\
\nu_{k} & =\frac{\sqrt{\tilde{\alpha}}}{\mu}\left(k^{2}-k_{R}^{2}\right)^{\frac{1}{2}} \\
\gamma_{k} & =\arg \left(\Gamma\left(-2 \nu_{k}\right)\left(e^{-2 \pi i \nu_{k}}-e^{-\frac{2 \pi}{\sqrt{3}} e_{R} \sqrt{\tilde{\alpha}}}\right)\right) .
\end{aligned}
$$

The coefficients $h_{1} \sim \frac{r_{-}}{R^{2}}$ and $h_{2} \sim\left(\frac{r_{-}}{R^{2}}\right)^{1-2 \nu_{k_{F}}}$ can be computed numerically. For $\nu_{k}>\frac{1}{2}, \nu_{k}=\frac{1}{2}$ or $\nu_{k}>\frac{1}{2}$ we have a Fermi liquid, a marginal Fermi liquid or a non-Fermi liquid, respectively. Note that the transition from a non-Fermi liquid to a Fermi liquid occurs for $k^{0} \approx k_{c}^{0}$ which is fixed by the condition $k_{c}^{0} \approx\left|v_{F} \Pi\left(k_{c}^{0}\right)\right|$. Below, we will refer to the non-Fermi liquid region $k^{0}<k_{c}^{0}$ as the near horizon or emergent contribution, and the Fermi liquid region $k^{0}>k_{c}^{0}$ as the far horizon or dilute contribution. The two contributions will be added approximately since an exact construction that interpolates between these two limiting regimes is likely numerical and outside the scope of this work.

A schematic description of the poles of (2.13) is given in Fig. 2. For sufficiently large effective charge $e_{R} \sqrt{\tilde{\alpha}}$, some of the largely damped quasinormal modes (QNM) of the RN-AdS black hole transmute to narrow quasi-bound states (QBS) close to the real axis for fixed $k<k_{F}$. For increasing $k \rightarrow k_{F}$ the narrow QBS start crossing the origin $\omega=0$ turning to equally spaced holographic Fermi surfaces (here 4 Fermi surfaces) as discussed in [19].

For fermions with larger effective charge, i.e., for $e_{R}^{2} \tilde{\alpha}>$ $\frac{1}{4}(m R)^{2}$ or $k_{R}^{2}>0$, pair creation takes place near the horizon as we noted earlier. A hallow of charged fermions at the Fermi surface with $k_{F}>k_{R}>0$, that supports quasiparticles with $\mathcal{G}_{F}^{11}$ given in (2.13). For hard R-probes with large $q^{0}$ in the DIS kinematics, only $\mathcal{G}_{R}^{11}\left(k^{0}, \vec{k}\right)$ is modified close to the horizon, since $\mathcal{G}_{R}^{11}\left(\omega_{1}, k+q\right)$ carries a large momentum and is mostly unmodified in the ultraviolet,

$$
\begin{aligned}
& \operatorname{Im}_{R}^{11}\left(\omega_{1}, k+q\right) \operatorname{Im} \mathcal{G}_{R}^{11}\left(k^{0}, \vec{k}\right) \\
& \rightarrow \operatorname{Tr}\left(\left(\sigma_{1}\left(k^{0}+q^{0}\right)-i \sigma_{2}\left(k_{x}+q_{x}\right)-\omega_{1}\right)\right. \\
& \left.\quad \times \pi \delta\left((k+q)^{2}+\omega_{1}^{2}\right) \operatorname{Im} \mathcal{G}_{R}^{11}\left(k^{0}, \vec{k}\right)\right)
\end{aligned}
$$

\section{HOLOGRAPHIC STRUCTURE FUNCTIONS}

The holographic structure functions on an extremal black hole in leading order have been discussed in [15], to which we refer for further details. For completeness, the results will be summarized below, and extended to allow for the next to leading order contributions from the holographic Fermi liquid at the horizon.

\section{A. Structure functions}

We recall that the scattering amplitude of an R-photon of longitudinal momentum $q^{\mu}=(\omega, q, 0,0)$ scattering on a black hole at rest in the Lab frame with $n^{\mu}=(1,0,0,0)$, (3.22), can be tensorially decomposed into two invariant functions $\tilde{G}_{1,2}[13]$

$$
\begin{aligned}
\tilde{G}_{\mu \nu}^{F}(q)= & \left(\eta_{\mu \nu}-\frac{q_{\mu} q_{\nu}}{Q^{2}}\right) \tilde{G}_{1}+\left[n_{\mu} n_{\nu}-\frac{n \cdot q}{Q^{2}}\left(n_{\mu} q_{\nu}+n_{\nu} q_{\mu}\right)\right. \\
& \left.+\frac{q_{\mu} q_{\nu}}{\left(Q^{2}\right)^{2}}(n \cdot q)^{2}\right] \tilde{G}_{2}
\end{aligned}
$$

with $Q^{2}=q^{2}$, thanks to with the current conservation and covariance. The corresponding DIS structure functions for an R-photon on a black hole are defined as

$$
\begin{aligned}
& \tilde{F}_{1}=\frac{1}{2 \pi} \operatorname{Im} \tilde{G}_{1}, \\
& \tilde{F}_{2}=-\frac{(n \cdot q)}{E_{A}} \operatorname{Im} \frac{1}{2 \pi} \tilde{G}_{2} .
\end{aligned}
$$

As in [13], the rest frame of a cold and extremal black hole will be dual to the rest frame of a cold nucleus at the boundary with fixed energy $E_{A}=\frac{3}{4} A \mu$. Since the binding 
energy in a nucleus is small, we also have $E_{A} \simeq A m_{N}$ and therefore the chemical potential $\mu \simeq \frac{4}{3} m_{N}$. In our mapping, $m_{N}$ and $\mu$ are interchangeable for estimates. A hard photon with virtual momentum $q^{\mu}=(\omega, q, 0,0)$ scattering off the nucleus in the DIS kinematics satisfies $q^{2}-\omega^{2} \equiv Q^{2} \rightarrow \infty$ with $\omega \simeq q$ and fixed Bjorken-X

$$
x_{A}=\frac{q^{2}}{-2 q \cdot\left(n E_{A}\right)} \equiv \frac{Q^{2}}{2 E_{A} \omega}=\frac{x m_{N}}{E_{A}}
$$

$x_{A}$ will refer to the Bjorken variable of the black hole as a nuclear target with $0 \leq x_{A} \leq 1$, and $x$ to the Bjorken variable of the free nucleon in the LAB frame with $0 \leq x \leq A$.

\section{B. Classical black hole in leading order: Small-x}

As we noted earlier, the leading order contribution to the structure functions (3.2) in DIS scattering is classical and of order $N_{c}^{2}$ as illustrated in Fig. 1. It does not involve scattering off the fermions near the holographic surface, which is of order $N_{c}^{0}$. In the regime $Q \ll q \ll Q^{2} / \mu$ the leading contribution to the structure functions vanishes, as the probe spin-1 R-field is prevented from falling to the black hole by an induced potential barrier [12]. The $\mathrm{R}$-current correlator is purely real with an exponentially vanishing imaginary part. In the regime $q \gg Q^{3} / \mu^{2}$, the barrier wanes away with the classical and leading contribution to the un-normalized structure function $\tilde{F}_{2}$ of the form [13]

$\tilde{F}_{2}\left(x_{A}, Q^{2}\right) \approx \tilde{C}_{T} \frac{\mu^{2}}{x_{A}}\left(\frac{x_{A}^{2} Q^{2}}{\mu E_{A}}\right)^{\frac{2}{3}}+\tilde{C}_{L} \frac{E_{A}}{\mu} \frac{\mu^{2}}{x_{A}}\left(\frac{x_{A}^{2} Q^{2}}{\mu E_{A}}\right)$

with

$$
\begin{aligned}
\tilde{C}_{T} & =\frac{N_{c}^{2}}{2^{17 / 3} \pi^{2} \Gamma^{2}(1 / 3) \tilde{\alpha}^{5 / 3}} \\
\tilde{C}_{L} & =\frac{N_{c}^{2}}{1152 \pi^{4} \tilde{\alpha}^{2}}
\end{aligned}
$$

with $x_{A} E_{A}=x m_{N}$ and $C_{L} \ll C_{T}$. This result was shown to hold for low-x or $x_{A} \ll \sqrt{\mu E_{A}} / Q$, with the Callan-Gross relationlike $\tilde{F}_{2}=2 x_{A} \tilde{F}_{1}$. The normalized structure functions follow as [13]

$$
F_{1,2} \equiv 2 E_{A} V_{A} \tilde{F}_{1,2} \equiv\left(\frac{12 \pi \tilde{\alpha} A}{N_{c} \mu}\right)^{2} \tilde{F}_{1,2}
$$

after using the black-hole equation of state. More specifically, we have $\left(Q^{2}=q^{2}>0\right)$

$$
\frac{F_{2}^{\mathrm{BH}}\left(x, q^{2}\right)}{A} \approx \frac{C_{T}}{x}\left(\frac{3 x^{2} q^{2}}{4 m_{N}^{2}}\right)^{\frac{2}{3}}+\frac{3 C_{L}}{4 x}\left(\frac{3 x^{2} q^{2}}{4 m_{N}^{2}}\right)
$$

with $C_{T, L} / \tilde{C}_{T, L}=\pi^{5}(48 \tilde{\alpha})^{2} / 2 N_{c}^{2}$.
The normalization in (3.6) amounts overall to normalizing $\tilde{F}_{1,2}$ by the density of the black hole, canceling part of the model dependence of the equation of state. In a way, the normalized $F_{1,2}$ are the un-normalized black-hole structure functions $\tilde{F}_{1,2}$ per degree of freedom. (3.7) is dominated by the first contribution at low-x. We now show that the next contribution is dominated by scattering off bulk fermions at large-x from a holographic Fermi liquid close to the horizon.

\section{Classical black hole in leading order: Large-x}

The large- $x$ contribution to the dense black hole can be obtained using the WKB approach also developed for the thermal black hole in [12] with similar results modulo pertinent changes in the parameters and normalization. In particular, the structure function for $x>\mu / q$ is found to drop exponentially with the result

$$
\frac{F_{2}^{\mathrm{BH}}\left(x, q^{2}\right)}{A} \approx \frac{3 C_{L}}{2 x}\left(\frac{3 x^{2} q^{2}}{4 m_{N}^{2}}\right) D(x),
$$

where to exponential accuracy

$$
D(x) \approx C_{D} e^{-\sqrt{\frac{q}{m_{N} \mu}} \sqrt{x}},
$$

with $C_{D} \approx e^{-\frac{2 \Gamma^{2}(1 / 4) \sqrt{6 \tilde{\alpha}}}{3 \sqrt{2 \pi}}}$. Below we will use (3.7) plus (3.8) to describe DIS scattering on the $\mathrm{BH}$ in leading order. We now proceed to analyze the quantum and subleading correction.

\section{Quantum fermions in subleading order}

The contribution of the subleading fermions to the induced effective action can be obtained through the holographic dictionary. It will be divided into two contributions: 1/ the one stemming from the emergent Fermi surface near the horizon through the geometrical reduction to $\mathrm{AdS}_{2} \times \mathrm{R}^{3} ; 2 /$ the one stemming from its ultraviolet completion which is dual to a Fermi liquid in bulk $\mathrm{AdS}_{5}$ which we will seek in the dilute approximation below.

With this in mind, the shift of the R-field $A_{M} \rightarrow A_{0} \delta_{M 0}+$ $a_{M}$ amounts to a shift in the Dirac action density in (2.8) at the origin of the minimal coupling of the R-field

$$
-i \bar{\psi}\left(-i e_{R} a_{\mu}(r, q) \Gamma^{\mu}\right) \psi \equiv \bar{\psi} B(r ; q) \psi
$$

In terms of (2.8)-(3.10) the bulk effective action for the 1-loop contribution in Fig. $1 \mathrm{~b}$ at zero temperature reads

$$
\begin{aligned}
\mathcal{S}_{F}\left[a_{\mu}\right]= & -(-i) \int \frac{d^{4} q}{(2 \pi)^{4}} \frac{d^{4} k}{(2 \pi)^{4}} \\
& \times \int d r_{1} \sqrt{g\left(r_{1}\right)} d r_{2} \sqrt{g\left(r_{2}\right)} \operatorname{Tr}\left(D_{F}\left(r_{1}, r_{2} ; k+q\right)\right. \\
& \left.\times B\left(r_{2} ; q\right) D_{F}\left(r_{2}, r_{1} ; k\right) B\left(r_{1} ; q\right)\right)
\end{aligned}
$$


The routing of the momenta in (3.11) corresponds to the hard fermion with $k+q$ and the soft fermion with $k$.

The R-field in bulk $a(r, q)$ relates to the R-field at the boundary $A_{\mu}^{(0)}(q)$ through the bulk-to-boundary propagator $K_{A}(r ; q)$, which satisfies $K_{A}(r \rightarrow \infty ; q)=1$,

$$
a_{\mu}(r ; q)=K_{A}(r ; q) A_{\mu}^{(0)}(q)
$$

This allows the rewriting of (3.11) in the form of the boundary action

$$
\begin{aligned}
\mathcal{S}_{F}\left[A_{\mu}^{(0)}\right]= & \frac{1}{2} \int \frac{d^{4} q}{(2 \pi)^{4}} A_{\mu}^{(0)}(q) A_{\nu}^{(0)}(-q) \\
& \times(-2 i) \int \frac{d^{4} k}{(2 \pi)^{4}} \int d r_{1} d r_{2} \sqrt{g\left(r_{1}\right)} \sqrt{g\left(r_{2}\right)} \operatorname{Tr}\left(D_{F}\left(r_{1}, r_{2} ; k+q\right) Q^{\mu}\left(r_{2} ; q\right) D_{F}\left(r_{2}, r_{1} ; k\right) Q^{\nu}\left(r_{1} ; q\right)\right)
\end{aligned}
$$

with the dressed bulk vertices

$$
\begin{aligned}
Q^{\mu}(r ; q) & =-i\left(-i e_{R} K_{A}(r ; q) \Gamma^{\mu}\right) \\
& \approx-e_{R} \frac{q R^{2}}{r} K_{1}\left(\frac{q R^{2}}{r}\right) \Gamma^{\mu} .
\end{aligned}
$$

We have approximated the bulk-to-boundary $K_{A}(r ; q)$ by its vacuum contribution, with $K_{1}(x)$ the modified Bessel function.

In the DIS regime $Q \ll q \ll Q^{2} / \mu$ with $Q^{2}=q^{2}$, the spin- $\frac{1}{2}$ fermion field remains localized near the boundary as a potential barrier develops in bulk, a phenomenon also observed for spin-1 boson fields [12]. In this regime, we will approximate the hard part of the fermion propagator by its vacuum (in $\mathrm{AdS}_{5}$ ) result [20]

$D_{F}\left(r_{1}, r_{2} ; k+q\right)=\int d \omega_{1} \omega_{1} G_{F}\left(r_{1}, r_{2} ; \omega_{1}, k+q\right)$,

where

$G_{F}\left(r, r^{\prime} ; \omega_{1}, k+q\right)=\boldsymbol{\psi}_{\alpha}\left(r, \omega_{1}\right) \mathcal{G}_{F}^{\alpha \gamma}\left(\omega_{1}, k+q\right) \overline{\boldsymbol{\Psi}_{\gamma}}\left(r^{\prime}, \omega_{1}\right)$

with the vacuum (in $\mathrm{AdS}_{5}$ ) solution [21]

$$
\begin{aligned}
& \boldsymbol{\psi}_{1}\left(r, \omega_{1}\right)=\left(\frac{R^{2}}{r}\right)^{\frac{5}{2}} J_{m R-\frac{1}{2}}\left(\omega_{1} \frac{R^{2}}{r}\right)\left(\begin{array}{l}
0 \\
1
\end{array}\right) \\
& \boldsymbol{\psi}_{2}\left(r, \omega_{1}\right) \equiv 0,
\end{aligned}
$$

and

$$
\begin{aligned}
& \mathcal{G}_{F}^{11}\left(\omega_{1}, k+q\right)=\frac{\sigma_{1}\left(k^{0}+q^{0}\right)-i \sigma_{2}\left(k_{x}+q_{x}\right)-\omega_{1}}{(k+q)^{2}+\omega_{1}^{2}-i \epsilon}, \\
& \mathcal{G}_{F}^{22}\left(\omega_{1}, k+q\right)=\frac{\sigma_{1}\left(k^{0}+q^{0}\right)+i \sigma_{2}\left(k_{x}+q_{x}\right)-\omega_{1}}{(k+q)^{2}+\omega_{1}^{2}-i \epsilon} .
\end{aligned}
$$

The soft part of the fermion propagator can be separated into its contribution deep in the infrared which is modified by the induced holographic Fermi surface through the geometrical reduction to $\mathrm{AdS}_{2} \times \mathrm{R}^{3}$, and its ultraviolet completion as we noted earlier. More specifically, near the $\mathrm{AdS}_{2} \times \mathrm{R}^{3}$ geometry, the infrared part of the soft the propagator is of the form

$D_{F}\left(r, r^{\prime} ; k\right)=\boldsymbol{\psi}_{\alpha}\left(r, k^{0}, \vec{k}\right) \mathcal{G}_{\alpha \beta}^{F}\left(k^{0}, \vec{k}\right) \overline{\boldsymbol{\Psi}_{\beta}}\left(r^{\prime}, k^{0}, \vec{k}\right)$

with

$\mathcal{G}_{F}^{22}\left(k^{0}, \vec{k}\right)=\mathcal{G}_{F}^{11}\left(k^{0}, \vec{k} \mapsto-\vec{k}\right)=-\frac{1}{\mathcal{G}_{F}^{11}\left(k^{0}, \vec{k} ; m \mapsto-m\right)}$.

Note that only $\mathcal{G}_{R}^{11}\left(k^{0}, \vec{k}\right)$ has a singular or Fermi-like structure near $k \rightarrow k_{F}$. Hence, we will ignore the contribution from $\mathcal{G}_{R}^{22}\left(k^{0}, \vec{k}\right)$ to the current correlator. The normalizable wave functions are given in (B1).

The time-ordered correlation function for the R-current follows from the functional derivative

$$
\tilde{G}^{F \mu \nu}(q)=\frac{\delta^{2} \mathcal{S}_{F}\left[A_{\mu}^{(0)}\right]}{\delta A_{\mu}^{(0)}(q) \delta A_{\nu}^{(0)}(-q)} .
$$

Using the spectral form of the Feynman propagator (3.19)(3.20), we can rewrite (3.21) in a more compact form

$$
\begin{aligned}
\tilde{G}^{F \mu \nu}(q)= & 2 i \int \frac{d^{4} k}{(2 \pi)^{4}} \int d \omega_{1} \omega_{1} \operatorname{Tr}\left(\mathcal{G}_{F}^{\alpha \beta}\left(\omega_{1}, k+q\right)\right. \\
& \left.\times \Lambda_{\beta \gamma}^{\mu}\left(\omega_{1} ; q ; k\right) \mathcal{G}_{F}^{\gamma \delta}\left(k^{0}, \vec{k}\right) \Lambda_{\delta \alpha}^{\nu}\left(k ; q ; \omega_{1}\right)\right),
\end{aligned}
$$

with the dressed vertices

$$
\Lambda_{\beta \gamma}^{\mu}\left(\omega_{1} ; q ; k\right)=\int d r_{2} \sqrt{g\left(r_{2}\right)} \overline{\boldsymbol{\Psi}_{\beta}}\left(r_{2}, \omega_{1}\right) Q^{\mu}\left(r_{2} ; q\right) \boldsymbol{\psi}_{\gamma}\left(r_{2}, k\right),
$$

and 
$\Lambda_{\delta \alpha}^{\nu}\left(k ; q ; \omega_{1}\right)=\int d r_{1} \sqrt{g\left(r_{1}\right)} \overline{\boldsymbol{\Psi}_{\beta}}\left(r_{1}, k\right) Q^{\nu}\left(r_{1} ; q\right) \boldsymbol{\Psi}_{\alpha}\left(r_{1}, \omega_{1}\right) . \quad \mathcal{G}_{F}\left(k^{0}, \vec{k}\right)=\operatorname{Re} \mathcal{G}_{R}\left(k^{0}, \vec{k}\right)+i \operatorname{sgn}\left(k^{0}\right) \operatorname{Im} \mathcal{G}_{R}\left(k^{0}, \vec{k}\right)$

We recall that at zero temperature, the general Feynman and retarded propagators $\mathcal{G}_{F, R}$ are related by the relationship

Using (3.25) and the fact that $\mathcal{G}_{F}\left(k^{0}, \vec{k}\right)$ is analytic in the upper complex $k^{0}$-plane, allow for the rewriting of the imaginary part of (3.22) in the form

$$
\begin{aligned}
\operatorname{Im} \tilde{G}_{\mu \nu}^{F}(q) & =\int \frac{d^{4} k}{(2 \pi)^{4}} \int d \omega_{1} \omega_{1} \Lambda_{\beta \gamma}^{\mu}\left(\omega_{1} ; q ; k\right) \Lambda_{\delta \alpha}^{\nu}\left(k ; q ; \omega_{1}\right) \operatorname{Re} \operatorname{Tr}\left(\mathcal{G}_{F}^{\alpha \beta}\left(\omega_{1}, k+q\right) \mathcal{G}_{F}^{\gamma \delta}\left(k^{0}, \vec{k}\right)\right), \\
& =\int \frac{d^{3} k}{(2 \pi)^{3}} \int_{-\left|q^{0}\right|}^{0} \frac{d k^{0}}{2 \pi} \int d \omega_{1} \omega_{1} \Lambda_{\beta \gamma}^{\mu}\left(\omega_{1} ; q ; k\right) \Lambda_{\delta \alpha}^{\nu}\left(k ; q ; \omega_{1}\right) \operatorname{Tr}\left(\operatorname{Im} \mathcal{G}_{R}^{\alpha \beta}\left(\omega_{1}, k+q\right) \operatorname{Im} \mathcal{G}_{R}^{\gamma \delta}\left(k^{0}, \vec{k}\right)\right),
\end{aligned}
$$

This result shows that for $q^{0}=0$, the imaginary part vanishes as it should as the effective action induced by the R-current (3.13) is real. For $q^{0} \neq 0$ this result is clearly negative as it should, since its contribution to (3.13) amounts to a self-energy for the R-field which amounts to damped oscillations in time.

\section{E. Large-x near the horizon}

Using the vertex (D5) for momenta near $k_{F}$, we can rewrite (3.26)

$$
\operatorname{Im} \tilde{G}_{x x}^{F}(q)=C^{2}\left(\nu_{k_{F}}\right) C_{\theta} k_{F}^{2}(-1) \int_{0}^{\infty} \frac{d \omega_{1}^{2}}{2} I_{z}^{2}\left(\omega_{1} ; q ; k_{F}\right) \int_{k_{R}}^{k_{F}} d k a_{+}\left(k_{0}, k\right)^{2} \operatorname{Re} I_{k^{0}}\left(\omega_{1}, q\right),
$$

with

$$
\begin{aligned}
\operatorname{ReI}_{k^{0}}\left(\omega_{1}, q\right) & =\operatorname{Re} \int_{-\infty}^{\infty} \frac{d k^{0}}{2 \pi} a_{+}\left(k_{0}, k\right)^{2} \operatorname{tr}\left(\mathcal{G}_{F}^{11}\left(\omega_{1}, k+q\right) \mathcal{G}_{F}^{11}\left(k^{0}, \vec{k}\right)\right) \\
& =\operatorname{Re} \int_{-\left|q^{0}\right|}^{0} \frac{d k^{0}}{2 \pi} a_{+}\left(k_{0}, k\right)^{2} \operatorname{tr}\left(\left(\sigma_{1}\left(k^{0}+q^{0}\right)-i \sigma_{2}\left(k_{x}+q_{x}\right)-\omega_{1}\right) \pi \delta\left((k+q)^{2}+\omega_{1}^{2}\right) \operatorname{Im} \mathcal{G}_{R}^{11}\left(k^{0}, \vec{k}\right)\right) \\
& \approx \int_{-\left|q^{0}\right|}^{0} \frac{d k^{0}}{2 \pi} a_{+}\left(k_{0}, k\right)^{2}(-1) \omega_{1} \pi \delta\left((k+q)^{2}+\omega_{1}^{2}\right) \frac{\mathrm{h}_{1} \operatorname{Im} \Pi}{\left(k-k_{F}-\frac{k^{0}}{v_{F}}-\operatorname{Re} \Pi\right)^{2}+(\operatorname{Im} \Pi)^{2}}
\end{aligned}
$$

(3.28) can be simplified by enforcing the delta function

$$
\begin{aligned}
\operatorname{Im} \tilde{G}_{x x}^{F}(q) \approx & C^{2}\left(\nu_{k_{F}}\right) C_{\theta} k_{F}^{2} \frac{\pi}{2} \int_{k_{R}}^{k_{F}} d k \int_{-\left|q^{0}\right|}^{0} \frac{d k^{0}}{2 \pi} a_{+}^{2}\left(k_{0}, k\right) I_{z}^{2}\left(x_{k} ; q ; k_{F}\right) \sqrt{s_{k}} \frac{h_{1} \operatorname{Im} \Pi}{\left(k-k_{F}-\frac{k^{0}}{v_{F}}-\operatorname{Re} \Pi\right)^{2}+(\operatorname{Im} \Pi)^{2}} \\
\approx & C_{G}\left(\nu_{k_{F}}, z_{-}\right)\left(\frac{1}{q^{2}}\right)^{\nu_{k_{F}}+\frac{3}{2}} \int_{k_{R}}^{k_{F}} d k k_{F}^{2} \int_{-\left|q^{0}\right|}^{0} \frac{d k^{0}}{2 \pi} \\
& \times a_{+}^{2}\left(k_{0}, k\right) x_{k}^{\nu_{k_{F}}+5 / 2}\left(1-x_{k}\right)^{m R-1 / 2}{ }_{2}^{2} F_{1}^{2}\left(\frac{m R+\nu_{k_{F}}+2}{2}, \frac{m R-\nu_{k_{F}}+1}{2}, m R+\frac{1}{2}, 1-x_{k}\right) \\
& \times \sqrt{s_{k}} \frac{\operatorname{Im} \Pi}{\left(k-k_{F}-\frac{k^{0}}{v_{F}}-\operatorname{Re} \Pi\right)^{2}+(\operatorname{Im} \Pi)^{2}}
\end{aligned}
$$

with the overall constant

$$
C_{G}\left(\nu_{k_{F}}, z_{-}\right)=\frac{\pi}{2} z_{-}^{-\left(2 \nu_{k}+2\right)} C^{2}\left(\nu_{k_{F}}\right) C_{z}^{2}\left(\nu_{k_{F}}\right) \tilde{h}_{1}\left(\nu_{k_{F}}\right) C_{\theta},
$$

where $\tilde{h}_{1}\left(\nu_{k_{F}}\right) \equiv z_{-} h_{1}$ is a dimensionless constant to be determined numerically, and $z_{-}=\frac{R^{2}}{r_{-}}$. Again, for the DIS kinematics we set $x_{k}=-q^{2} / 2 k \cdot q$, and $\left|q^{0}\right| \approx q_{x}$. We rearranged the hypergeometric function ${ }_{2} F_{1}$ using the same Pfaff identity (3.36). Note that for the special value of $\nu_{k}=m R+1$, one can see that the $x_{k}$ dependence of the integrand in (3.29) reduces to the one in [21] before the multiplication by the trace (for our case the trace is $\sqrt{s_{k}}$ ). However, for general $\nu_{k}$ the same partonic content as in (3.37) is noted. 
For narrow quasiparticles, we may use the substitution $\left(k_{\perp}=k-k_{F}\right)$

$$
\frac{\operatorname{Im} \Pi}{\left(k_{\perp}-\frac{k^{0}}{v_{F}}-\mathrm{Re} \Pi\right)^{2}+(\operatorname{Im} \Pi)^{2}} \rightarrow \pi \delta\left(k_{\perp}-\frac{k^{0}}{v_{F}}-\mathrm{Re} \Pi\right)
$$

and undo the $k^{0}$ integration in (3.29) with the result

$$
\begin{aligned}
\operatorname{Im} \tilde{G}_{x x}^{F}(q) \approx & \frac{1}{2} \tilde{C}_{G}\left(\nu_{k_{F}}\right) \int_{k_{R}}^{k_{F}} d k_{\perp} k_{F}^{2} \frac{a_{+}\left(k_{0}, k_{\perp}\right)^{2}}{\left|\frac{1}{v_{F}}+\operatorname{Re} \Pi^{\prime}\right|}\left(\frac{1}{q^{2} z_{-}^{2}}\right)^{\nu_{k_{F}}+1} \\
& \times x_{k}^{\nu_{k_{F}}+2}\left(1-x_{k}\right)^{\tau-\frac{3}{2}}{ }_{2} F_{1}^{2}\left(\tau_{+}, \tau_{-}, \tau-1,1-x_{k}\right)
\end{aligned}
$$

with

$$
\operatorname{Re} \Pi^{\prime}=2 h_{2} \nu_{k_{F}}\left|k^{0}\right|^{2 \nu_{k_{F}}-1} \operatorname{Re}\left(e^{i \gamma_{k_{F}}}(-1)^{2 \nu_{k_{F}}-1}\right)
$$

where $k^{0}$ in $x_{k}$ is solution to the transcendental equation

$$
k_{\perp}+\frac{\left|k^{0}\right|}{v_{F}}=h_{2}\left|k^{0}\right|^{2 \nu_{k_{F}}} \operatorname{Re}\left(e^{i \gamma_{k_{F}}}(-1)^{2 \nu_{k_{F}}}\right)
$$

and we have defined a dimensionless constant

$$
\begin{aligned}
\tilde{C}_{G}\left(\nu_{k_{F}}\right) & =z^{2 \nu_{k_{F}}+2} \times C_{G}\left(\nu_{k_{F}}, z_{-}\right) \\
& =\frac{\pi}{2} C^{2}\left(\nu_{k_{F}}\right) C_{z}^{2}\left(\nu_{k_{F}}\right) \tilde{h}_{1}\left(\nu_{k_{F}}\right) C_{\theta} .
\end{aligned}
$$

Also note that $a_{+}\left(k_{0}, k_{\perp}\right)=\bar{c}_{1} z_{-} k_{\perp}+\bar{c}_{2} z_{-} k_{0}$ is a dimensionless coefficient with dimensionless constants $\bar{c}_{1,2}=$ $\tilde{c}_{1,2} / z_{-}$.

In arriving to (3.32), we have made use of the Pfaff identity

$$
{ }_{2} F_{1}(a, b, c, z)=(1-z)^{-a}{ }_{2} F_{1}\left(a, c-b, c, \frac{z}{z-1}\right)
$$

In the holographic Fermi liquid, the partonic distribution function is seen to develop a modified exponent. A comparison of the partonic distribution function (6.13) in the probe limit, to $(\mathrm{F} 4)$ where the black hole is present, translates

$$
\begin{aligned}
& \left(\frac{1}{q^{2}}\right)^{\tau-1} x_{k}^{\tau+1}\left(1-x_{k}\right)^{\tau-2} \\
& \rightarrow\left(\frac{1}{q^{2}}\right)^{\nu_{k}+1} x_{k}^{\nu_{k}+2}\left(1-x_{k}\right)^{\tau-\frac{3}{2}}{ }_{2} F_{1}^{2}\left(\tau_{+}, \tau_{-}, \tau-1,1-x_{k}\right)
\end{aligned}
$$

with $2 \tau_{ \pm}=\tau \pm\left(\nu_{k}+1 / 2\right)$ and the twist parameter $\tau=m R+3 / 2$. Near the black hole horizon, the parton distribution function develops a modified scaling law, but it

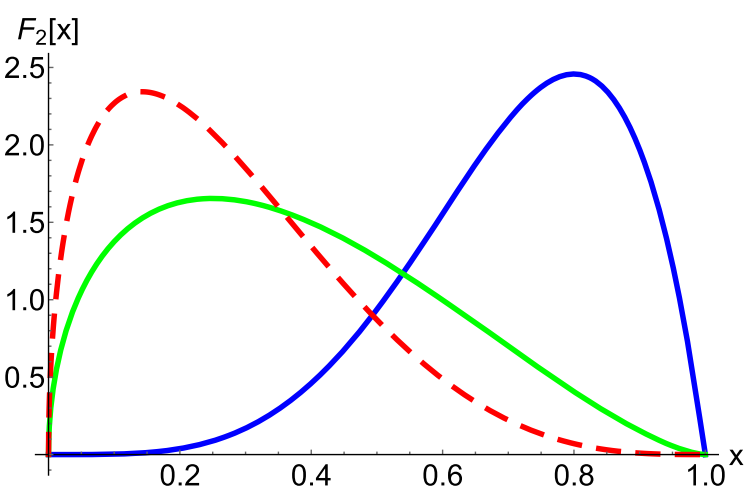

FIG. 3. Large-x dependence of the parton distribution function for weak coupling in vacuum (dashed-red), strong coupling in vacuum (dark-solid-blue) and near a holographic Fermi surface (light-solid-green).

is still seen to vanish at the end points $x_{k}=0$, 1. In Fig. 3 we show the modified behavior of the partonic distribution function in (3.37) for fixed $q^{2}, \tau=3$ and $\nu_{k}=\frac{1}{2}$ versus $x_{k}$ as the light-solid curve (green). The comparison is with the large-x dependence of the nucleon for weak coupling dashed curve (red), and strong coupling dark-solid curve (blue). Near the black hole horizon, the distribution function is shifted to intermediate-x. With our choice of parameters, the holographic result (3.37) reduces to $x_{k}^{\frac{1}{2}}\left(1-x_{k}\right)^{\frac{3}{2}}$, in comparison to the strong coupling result in vacuum $x_{k}^{4}\left(1-x_{k}\right)^{2}$, and the weak coupling result also in the vacuum $x_{k}^{\frac{1}{2}}\left(1-x_{k}\right)^{3}$. Remarkably, the formation of a holographic fermionic surface through the $\mathrm{AdS}_{2} \times \mathrm{R}^{3}$ reduction, is to shift the holographic partonic distribution to intermediate- $\mathrm{x}$, and modify the hard scattering rule.

For our choice of DIS kinematics, the non-normalized $\tilde{F}_{2}$ structure function (3.2) follows from (3.32) in the form $\left(q_{0} \approx q_{x}\right)$

$$
\tilde{F}_{2}\left(x_{A}, q\right)=\frac{4}{\pi} x_{A}^{3} \frac{E_{A}^{2}}{q^{2}} \operatorname{Im} \tilde{G}_{x x}^{F}(q)
$$

with again $Q^{2}=q^{2}>0$. Modulo the dispersion relation and the anomalous exponents that characterize the holographic fermions in the reduced $\mathrm{AdS}_{2} \times \mathrm{R}^{3}$ geometry, the results (3.32) and (3.38) are similar to the ones we derived recently in [15] using general arguments.

\section{FERMIONIC CONTRIBUTION AT LOW-X}

In the DIS regime with $q \gg Q^{2}$ or low-x, the structure functions are dominated by the exchange of a Pomeron, a multigluon exchange with vacuum quantum numbers. In holography, this exchange is described either through a closed surface exchange [22] or a graviton [23] in bulk. For the latter, this regime was identified in the range $e^{-\sqrt{\lambda}} \ll x \ll 1 / \sqrt{\lambda}$ where the exchange involves the string 
scattering amplitude. Since $x \gg e^{-\sqrt{\lambda}}$, the strings are small compared to the size of the AdS space so that the scattering amplitude is quasilocal with almost flat space signature.

\section{A. General setup}

The 10-dimensional tree-level effective action that describes the scattering of an R-photon off bulk quantum fermions at low-x reads [23]

$$
\begin{aligned}
\mathcal{S} & =\int d^{10} x \sqrt{-g_{10}}(\mathcal{K} V)_{t=0} \\
& =\left.\frac{i}{8} \int d^{10} x \sqrt{-g_{10}}\left(4 v^{a} v_{a} \bar{\psi} \Gamma_{m} \partial^{p} \psi-g_{m}^{p}\left(\bar{\psi} \Gamma^{M} \partial_{M} \psi v_{a} v^{a}+2 v_{a} v_{b} \bar{\psi} \Gamma^{a} \partial^{b} \psi\right)\right) F^{m n} F_{p n} \mathcal{V}\right|_{t=0}
\end{aligned}
$$

where $v^{a}$ are the Killing vectors for the compact part of the 10-dimensional space. The forward R-current scattering amplitude follows from pertinent variation with respect to the R-field. Here $\mathcal{K}$ refers to the kinematical factor involving the fermions $\psi$ and the R-field strength $F$, and $\mathcal{V}$ is the exchanged flat space 10-dimensional VirazorroShapiro string amplitude

$$
\mathcal{V}=\frac{\alpha^{\prime 3} \tilde{s}^{2}}{64} \prod_{\tilde{\beta}=\tilde{s}, \tilde{u}, \tilde{u}} \frac{\Gamma\left(-\frac{\alpha^{\prime} \tilde{\beta}}{4}\right)}{\Gamma\left(1+\frac{\alpha^{\prime} \tilde{\beta}}{4}\right)}
$$

as illustrated in Fig. 4. The 10-dimensional Mandelstam variables $\tilde{s}, \tilde{t}$ are related to the 4-dimensional ones $s, t$ through

$$
\begin{aligned}
& \alpha^{\prime} \tilde{s}=\alpha^{\prime} s \frac{z^{2}}{R^{2}}+\mathcal{O}\left(\frac{1}{\sqrt{\lambda}}\right) \\
& \alpha^{\prime} \tilde{t}=\alpha^{\prime} t \frac{z^{2}}{R^{2}}+\mathcal{O}\left(\frac{1}{\sqrt{\lambda}}\right)
\end{aligned}
$$

with the warping made explicit.

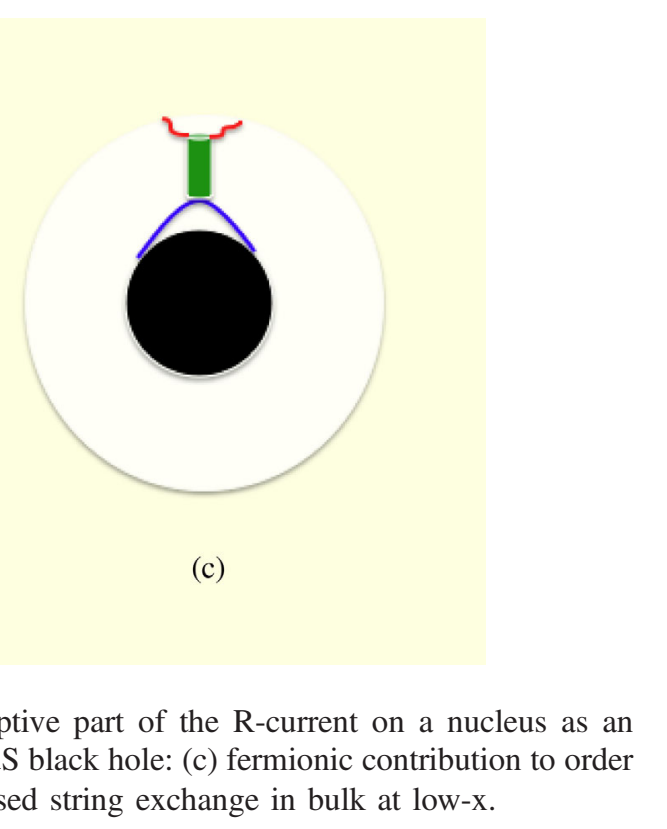

FIG. 4. Absorptive part of the R-current on a nucleus as an extremal RN-AdS black hole: (c) fermionic contribution to order $N_{c}^{0}$ due to a closed string exchange in bulk at low-x.
The imaginary part of the string amplitude (4.2) is

$$
\left.\operatorname{Im} \mathcal{V}\right|_{t=0}=\frac{\pi \alpha^{\prime}}{4} \sum_{j=1}^{\infty} j^{\mathcal{O}\left(\frac{1}{\sqrt{\lambda}}\right)} \delta\left(j-\frac{\alpha^{\prime} \tilde{s}}{4}\right)
$$

with the delta-function summing over the closed string Regge trajectory. At low-x we have $s \sim 1 / x$ and $j \sim s \sim$ $1 / x$, so that for $\ln (1 / x) / \sqrt{\lambda} \ll 1$,

$$
j^{\mathcal{O}\left(\frac{1}{\sqrt{\lambda}}\right)} \sim\left(\frac{1}{x}\right)^{\mathcal{O}\left(\frac{1}{\sqrt{\lambda}}\right)} \sim 1
$$

We now recall that the field strength $F^{m n}$ describes the bulk-to-boundary R-field-strength with incoming momentum $q^{\mu}$ and outgoing momentum $q^{\mu}$, while $\psi$ describes the bulk fermion with incoming and outgoing momentum $k^{\mu}$ in the anomalous Fermi surface. The low-x regime with $x \ll 1$ corresponds to the kinematical regime $q \cdot k \gg q^{2} \gg k^{2}$, so that the dominant contribution in $\mathcal{K}$ is the term with the spin contraction of the form $(q \cdot k)$, i.e., the first term in (4.1). Using

$$
\psi(k) \rightarrow \psi(k) \times \mathbb{Y}(v)
$$

and normalizing

$$
\int_{S^{5}} d \hat{v} \sqrt{g_{S^{5}}} v^{a} v_{a}|\bigvee(v)|^{2}=c_{5} R^{2}
$$

where $\mathbb{Y}(v)$ is a spherical harmonic in $S^{5}$ in (4.1), we can write down the one-loop effective action $\mathcal{S}_{F}$ for the diagram shown in Fig. 4 as

$$
\begin{aligned}
\mathcal{S}_{F}\left[A_{\mu}^{(0)} \equiv n_{\mu}\right]= & \frac{i}{2} c_{5} R^{2} \int \frac{d^{4} q}{(2 \pi)^{4}} \int \frac{d^{4} k}{(2 \pi)^{4}} \\
& \times \int d r \sqrt{-g} F^{\mu m}(-q) F_{\nu m}(q) \\
& \times\left.\operatorname{Tr}\left(D_{F}(r, r, k) \Gamma_{\mu}(-i k)^{\nu}\right) \mathcal{V}_{F}\right|_{t=0} .
\end{aligned}
$$

We now choose the polarizations to be transverse with the additional axial gauge condition $a_{r}=0$, so that the boundary-to-bulk R-field is 


$$
a_{\mu}(r, \vec{q})=\left(\frac{R^{2}}{r} K_{1}\left(\frac{q R^{2}}{r}\right)\right) n_{\mu}(q) e^{i q \cdot x}
$$

The corresponding field strengths are

$$
\begin{aligned}
& F_{\mu \nu}(q)=i\left(q_{\mu} n_{\nu}-n_{\mu} q_{\nu}\right) \frac{q R^{2}}{r} K_{1}\left(\frac{q R^{2}}{r}\right) e^{i q \cdot x}, \\
& F_{\mu r}(q)=n_{\mu} q^{2} \frac{R^{4}}{r^{3}} K_{0}\left(\frac{q R^{2}}{r}\right) e^{i q \cdot x}
\end{aligned}
$$

and their contraction is

$$
\begin{aligned}
F_{\mu p} F_{\nu}{ }^{p}= & +n_{\mu} n_{\nu} \frac{q^{4} R^{6}}{r^{4}}\left(K_{0}^{2}\left(\frac{q R^{2}}{r}\right)+K_{1}^{2}\left(\frac{q R^{2}}{r}\right)\right) \\
& +q_{\mu} q_{\nu} n^{2} \frac{q^{2} R^{6}}{r^{4}} K_{1}^{2}\left(\frac{q R^{2}}{r}\right) .
\end{aligned}
$$

\section{B. Low-x near the horizon}

To analyze the low-x contribution of the fermions near the horizon, we will focus on the graviton exchange and make use of warped momenta $\tilde{q}$ throughout this section. For small energy transfer $\tilde{q}_{0} \ll \mu$, the bulk-to-bulk propagator for transverse graviton $h_{x}{ }^{y}\left(\tilde{q}_{0}, \tilde{q}_{x}\right)$ can be written as

$$
\begin{aligned}
& G_{x y, x y}\left(\tilde{q}_{0}, \tilde{q}_{x}, r_{1}, r_{2}\right) \\
& \quad=\phi\left(\tilde{q}_{0}, \tilde{q}_{x}, r_{1}\right) G_{x y, x y}^{B}\left(\tilde{q}_{0}, \tilde{q}_{x}\right) \phi\left(q_{0}, q_{x}, r_{2}\right)
\end{aligned}
$$

where $\phi\left(\tilde{q}_{0}, \tilde{q}_{x}, r_{1}\right)$ is the normalizable wave function of the graviton, and $G_{x y, x y}^{B}$ is its boundary Green's function

$$
G_{x y, x y}^{B}\left(\tilde{q}_{0}, \tilde{q}_{x}\right)=\tilde{q}_{0}^{2} G\left(\tilde{q}_{0}, \tilde{q}_{x}\right)
$$

where $\operatorname{Re} G\left(\tilde{q}_{0}, \tilde{q}_{x}\right)=f\left(\tilde{q}_{x}, \mu\right)$ which can be determined from the low-frequency expansion, and [24]

$$
\begin{aligned}
\operatorname{Im} G\left(\tilde{q}_{0}, \tilde{q}_{x}\right)= & \frac{3 C}{\left(1+\frac{\tilde{q}_{0}^{2}}{\mu^{2}}\right)^{\frac{1}{2}}}\left(1+\left(1+\frac{\tilde{q}_{0}^{2}}{\mu^{2}}\right)^{\frac{1}{2}}\right) \\
& \times e_{0}\left(\frac{\tilde{q}_{x}}{\mu}\right) \operatorname{Im} \mathcal{G}_{ \pm}\left(\frac{\tilde{q}_{0}}{\mu}, \frac{\tilde{q}_{x}}{\mu}\right)
\end{aligned}
$$

where $C$ is a proportionality constant, $e_{0}\left(\frac{\tilde{q}_{x}}{\mu}\right)$ is a function to be determined from the low-frequency expansion coefficients, and

$\mathcal{G}_{ \pm}\left(\frac{\tilde{q}_{0}}{\mu}, \frac{\tilde{q}_{x}}{\mu}\right)=-2 \nu_{ \pm} e^{-i \pi \nu_{ \pm}} \frac{\Gamma\left(1-\nu_{ \pm}\right)}{1+\nu_{ \pm}}\left(\frac{1}{2} \frac{\tilde{q}_{0}}{\mu}\right)^{2 \nu_{ \pm}}$,

where

$$
\nu_{ \pm}=\frac{1}{2}\left(5+2 \frac{\tilde{q}_{x}^{2}}{\mu^{2}} \pm 4\left(1+\frac{\tilde{q}_{x}^{2}}{\mu^{2}}\right)^{\frac{1}{2}}\right)^{\frac{1}{2}}
$$

Note that for zero energy and momentum transfer ( $\tilde{q}_{0}=0$ and $\tilde{q}_{x}=0$ ), the bulk-to-bulk propagator of the graviton exchange vanishes $\operatorname{Im} G_{x y, x y}\left(0,0, r_{1}, r_{2}\right)=0$ since $\mathcal{G}_{ \pm}(0,0)=0$. Therefore, the t-channel contribution of the graviton for the current-current correlation function or forward deeply virtual Compton scattering away from the probe limit vanishes. Its Reggeized form through higher spins (closed string exchange), vanishes as well.

\section{R-RATIO FOR A QUANTUM CORRECTED RN-ADS BLACK HOLE}

\section{A. Particle and energy density at the horizon}

Having assessed the structure functions both at large- $x$ and small-x near the black hole horizon, we now need to normalize them. For that we need to evaluate the contribution of the bulk fermions near the horizon to the particle and energy densities, much like we did in the probe limit. More specifically, we define

$$
\begin{aligned}
n e_{R} & =\left\langle J^{t}\right\rangle(q z \ll 1) \\
\epsilon & =\left\langle T^{t t}\right\rangle(q z \ll 1)
\end{aligned}
$$

as the boundary expectation values of the time-component of the R-current and the energy momentum tensor. The expectation values follow from the holographic correspondence in the tadpole approximation in $\mathrm{AdS}$ as

$$
\begin{aligned}
\left\langle J^{t}\right\rangle= & -i e_{R} \int \frac{d^{3} k}{(2 \pi)^{3}} \int \frac{d k^{0}}{2 \pi} I_{K}(q z \ll 1) \\
& \times \overline{\psi_{1}}(k) \Gamma^{t} \psi_{1}(k) \operatorname{Im} \operatorname{Tr} \mathcal{G}_{R}^{11}\left(k^{0}, \vec{k}\right) \\
\left\langle T^{t t}\right\rangle= & \int \frac{d^{3} k}{(2 \pi)^{3}} \int \frac{d k^{0}}{2 \pi} I_{K}^{\prime}(q z \ll 1) \\
& \times \overline{\psi_{1}}(k) \Gamma^{t} \psi_{1}(k)\left(-i k^{0}\right) \operatorname{Im} \operatorname{Tr}_{\mathcal{G}_{R}^{11}}\left(k^{0}, \vec{k}\right)
\end{aligned}
$$

with $I_{K}^{\prime}(q z \ll 1) \approx I_{K}(q z \ll 1)$ playing the role of a spectral weight, and defined in (6.23). Evaluating the momentum integral near the Fermi surface, we find

$$
n=\frac{\left\langle J^{t}\right\rangle}{e_{R}} \approx C_{J} h_{1} C_{\theta} \frac{k_{F}^{3}\left(1-\frac{k_{R}}{k_{F}}\right)}{\left|\frac{1}{v_{F}}+\operatorname{Re} \Pi^{\prime}\right|}
$$

with $C_{\theta}=\frac{1}{2 \pi}$ and the dimensionless constant

$$
\begin{aligned}
C_{J}= & R^{4}\left(m R_{2}+\nu_{k_{F}}\right)\left(\frac{R_{2}}{R} k_{F} z_{-}+e_{R} \sqrt{\frac{\tilde{\alpha}}{3}}\right) \\
& \times \frac{a_{+}^{2}\left(k_{0}, k\right)}{\left(2 \nu_{k}+1\right) W^{2}}(2 \sqrt{3})^{-2 \nu_{k_{F}}} .
\end{aligned}
$$


Since $I_{K}=I_{K}^{\prime}$, we have $\epsilon=n k^{0}$. Note that $k_{0}$ is the solution of the transcendental equation (3.34), which near the Fermi surface $k \rightarrow k_{F}$ can be solved as $k_{0} \sim C_{0} / z_{-}$with the dimensionless constants

$$
C_{0}\left(\nu_{k_{F}}\right) \equiv\left(v_{F} \tilde{h}_{2} \operatorname{Im}\left(e^{i \gamma_{F}}(-1)^{2 \nu_{k_{F}}}\right)\right)^{\frac{1}{1-2 \nu_{k_{F}}}}
$$

and $\tilde{h}_{2}=z^{1-2 \nu_{k_{F}}} h_{2}$. Therefore, for the dense limit near the horizon, we make the identification

$$
E_{A} \equiv V_{A} \epsilon=A \epsilon / n=A k^{0}
$$

Here $n$ and $\epsilon$ are identified with the particle and energy densities of the target of volume $V_{A}$ and total energy $E_{A}$ following the identification in IIIA.

\section{B. Normalized structure functions: Dense regime}

Having determined $n, \epsilon$ in the dense limit near the horizon, we can now normalize the corresponding structure function (3.32) through the substitution

$\operatorname{Im} \tilde{G}_{x x}^{F}(q) \rightarrow 2 E_{A} V_{A} \times \operatorname{Im} \tilde{G}_{x x}^{F}(q)=2 E_{A} A \frac{\operatorname{Im} \tilde{G}_{x x}^{F}(q)}{n}$.

The integral in (3.32) can be evaluated near the Fermi surface $k \rightarrow k_{F}$ with the result

$$
\operatorname{Im} \tilde{G}_{x x}^{F}(q) \approx \frac{1}{2} \tilde{C}_{G}\left(\nu_{k_{F}}\right) a_{+}\left(k_{0}, k_{\perp}=0\right)^{2} \frac{k_{F}^{3}\left(1-\frac{k_{R}}{k_{F}}\right)}{\left|\frac{1}{v_{F}}+\operatorname{Re} \Pi^{\prime}\right|}\left(\frac{1}{q^{2} z_{-}^{2}}\right)^{\nu_{k_{F}}+1} x_{k_{F}}^{\nu_{k_{F}}+2}\left(1-x_{k_{F}}\right)^{\tau-\frac{3}{2}}{ }_{2} F_{1}^{2}\left(\tau_{+}, \tau_{-}, \tau-1,1-x_{k_{F}}\right) .
$$

Here $\tilde{C}_{G}\left(\nu_{k_{F}}\right)$ and $a_{+}\left(k_{0}, k_{\perp}=0\right) \approx \bar{c}_{2} z_{-} k_{0}$ are both dimensionless constants, and $x_{k_{F}} \approx \frac{E_{A}}{k_{0}} x_{A}=\frac{m_{N}}{k_{0}} x$ since $\frac{x_{A}}{x}=\frac{m_{N}}{E_{A}}$. Here $k_{0} \sim C_{0} / z_{-}$plays the role of the Fermi energy of the quasiparticles in the holographic Fermi liquid near the horizon $z_{-}$. We recall that

$$
\frac{1}{z_{-}}=\frac{r_{-}}{R^{2}}=\frac{1}{2 \sqrt{3}} \frac{\mu}{\sqrt{\tilde{\alpha}}}=\frac{R_{2}}{R} \frac{\mu}{\sqrt{\tilde{\alpha}}}
$$

with $\tilde{\alpha}=1$ for a $\mathrm{U}(1) \mathrm{R}$-charge, and $\tilde{\alpha}=\frac{1}{4} \frac{N_{c}}{N_{f}}$ for a D3-D7 U(1) vector charge.

Using (3.38) together with (5.7)-(5.8), we can extract the normalized structure function of the holographic Fermi liquid $\left(x_{k_{F}} k_{0}=x m_{N}\right)$

$$
\frac{F_{2}\left(x_{A}, q^{2}\right)}{A}=e_{R}^{2} C_{\mathrm{AdS} 2}\left(e_{R}, \tau, \tilde{\alpha}, v_{F}, \tilde{h}_{2}\right)\left(\frac{\mu^{2}}{q^{2}}\right)^{\nu_{k_{F}}+2} x_{k_{F}}^{\nu_{k_{F}}+5}\left(1-x_{k_{F}}\right)^{\tau-\frac{3}{2}}{ }_{2} F_{1}^{2}\left(\tau_{+}, \tau_{-}, \tau-1,1-x_{k_{F}}\right),
$$

where we defined the dimensionless constant

$$
C_{\mathrm{AdS} 2}\left(e_{R}, \tau, \tilde{\alpha}, v_{F}, \tilde{h}_{2}\right) \equiv\left(\frac{1}{3 \tilde{\alpha}}\right)^{\nu_{k_{F}}+2} \frac{1}{8} C_{0}^{3} \frac{\left(2 \nu_{k_{F}}+1\right)\left(\frac{1}{2 \sqrt{3}} \tau+\nu_{k_{F}}-\frac{\sqrt{3}}{4}\right) \Gamma\left(\tau+\nu_{k_{F}}+\frac{3}{2}\right)^{2} \Gamma\left(\tau+\nu_{k_{F}}-\frac{1}{2}\right)^{2}}{\left(\frac{k_{F}}{\mu} \sqrt{\tilde{\alpha}}+\frac{1}{\sqrt{3}} e_{R} \sqrt{\tilde{\alpha}}\right) \Gamma(\tau-1)^{2}},
$$

with

$$
\begin{aligned}
\nu_{k_{F}}\left(e_{R}, \tau, \tilde{\alpha}\right) & =\left(\frac{1}{12}\left(\tau-\frac{3}{2}\right)^{2}+\frac{k_{F}^{2}}{\mu^{2}} \tilde{\alpha}-\frac{1}{3} e_{R}^{2} \tilde{\alpha}\right)^{\frac{1}{2}} \\
C_{0} & =\left(v_{F} \tilde{h}_{2} \sin \left(\gamma_{F}+2 \pi \nu_{k_{F}}\right)\right)^{\frac{1}{1-2 \nu_{k_{F}}}} \\
\gamma_{F} & =\arg \left(\Gamma\left(-2 \nu_{k_{F}}\right)\left(e^{-2 \pi i \nu_{k_{F}}}-e^{-\frac{2 \pi}{\sqrt{3}} e_{R} \sqrt{\tilde{\alpha}}}\right)\right) .
\end{aligned}
$$

Note that for a large effective charge $e_{R} \sqrt{\tilde{\alpha}} \rightarrow \infty$, we have $\gamma_{F}=-2 \pi \nu_{k_{F}}$ and $C_{0} \rightarrow 0$ which implies that the structure function (5.10) vanishes in the probe limit $\tilde{\alpha} \sim \frac{N_{c}}{N_{f}} \rightarrow \infty$, which is also the regime where the backreaction from the flavor branes can be ignored.

\section{R-ratio in the dense regime}

The R-ratio for the quantum corrected black hole consists of: (1) the leading classical contributions (3.7) plus (3.8) both at small and large $x$ respectively; (2) the subleading quantum correction from the emergent Fermi surface (6.28) in the $\mathrm{AdS}_{2} \times \mathrm{R}^{3}$ in the near horizon approximation; (3) the subleading quantum correction from a normal Fermi liquid far from the horizon in the dilute or probe approximation (6.30) to be detailed below. To map this ratio on that of a dense nucleus, we follow [13] and define

$$
R_{\text {dense }}\left(x, q^{2}\right) \equiv \frac{\frac{1}{A} F_{2}^{\text {dense }}\left(x, q^{2}\right)}{F_{2}^{\text {nucleon }}\left(x, q^{2}\right)}
$$


with the dense structure function

$$
\begin{aligned}
\frac{F_{2}^{\text {dense }}\left(x, q^{2}\right)}{A} \approx & \left(C_{T}\left(\frac{3 q^{2}}{4 m_{N}^{2}}\right)^{\frac{2}{3}} x^{\frac{1}{3}}+\frac{3 C_{L}}{2 x}\left(\frac{3 x^{2} q^{2}}{4 m_{N}^{2}}\right) D(x)\right) \\
& +\left(C_{\mathrm{AdS} 2} e_{R}^{2}\left(\frac{\mu^{2}}{q^{2}}\right)^{\nu_{k_{F}}+2} x_{k_{F}}^{\nu_{k_{F}}+5}\left(1-x_{k_{F}}\right)^{\tau-\frac{3}{2}} F_{1}^{2}\left(\tau_{+}, \tau_{-}, \tau-1,1-x_{k_{F}}\right)+\mathbb{C}_{1} \frac{F_{2}^{\mathrm{AdS} 5}\left(x, q^{2}\right)}{A}\right),
\end{aligned}
$$

normalized by the nucleon structure function (6.31). For clarity, we recap the different definitions used for parton-x $\left(0 \leq x_{A} \leq 1\right)$ and entering (5.14) and the normalization $(6.31)$

$$
\begin{aligned}
x_{A} E_{A} & =x m_{N} \\
x m_{N} & \approx x_{k_{F}} k_{0} \equiv x_{k_{F}} k_{F}
\end{aligned}
$$

$x_{A}$ refers to the parton fraction in a nucleus, $x$ refers to the parton fraction in a nucleon within a nucleus, and $x_{k_{F}}$ refers to the parton fraction in relation to $k_{0} \sim C_{0} / z_{-}$from the emergent Fermi energy.

In (5.14), the first bracket is the leading and classical contribution, and the second bracket is the subleading and quantum contribution. More specifically, the first (3.7) (low$\mathrm{x}$ ) and second (3.8) (large-x) contributions stem from DIS scattering on the bulk classical black hole. The third (6.28) contribution stems from DIS scattering off the emerging holographic liquid close to the black hole horizon. The fourth and last contribution $\frac{F_{2}^{\text {Ads }}\left(x, q^{2}\right)}{A} \equiv \frac{F_{2}^{\text {dilute }}\left(x, q^{2}\right)}{A}$ (6.30) stems from DIS scattering off the distorted Fermi liquid far from the horizon in the probe approximation to be discussed thoroughly below. The quantum correction near the black hole is vanishingly small at small-x.

The relative and arbitrary normalization $\mathbb{C}_{1}=0.07$ is introduced to account for the normal Fermi liquid contribution far from the horizon which is asymptotically $\mathrm{AdS}_{5}$ as we discussed earlier. It will be estimated in the dilute limit below and added to the near horizon contribution. A more quantitative calculation using the exact response function throughout the holographic space distorted by the RN-AdS extremal black hole, that reduces to $\mathrm{AdS}_{2}$ near the horizon and asymptotes the dilute limit near the boundary, is numerically intensive and goes outside the scope of this work. We note that the values of $\mathbb{C}_{1} \leq 0.07$ keep the $\mathrm{AdS}_{2}$ plus $\mathrm{AdS}_{5}$ quantum corrections subleading in comparison to the leading RN-AdS black hole contribution for all values of $k_{F}$ and most parton-X.

To quantify each of the contributions in the R-ratio, we now need to fix the parameters entering this expression, many of which are tied by holography. We first fix the explicit holographic parameters: $\tilde{\alpha}=N_{c} / 4 N_{f}=1$ (ratio of branes), $2 \pi^{2} c_{5} / \sqrt{4 \pi \lambda}=0.01$ (strong coupling) and $e_{R}=$ 0.3 (charge of the probe fermions). Next, we fix the scaling parameters entering in the nucleon pdf: $\tau=3$ (hard scaling law) and $j=0.08$ (Pomeron intercept). The nucleon confining scale enters through $\beta=1 /\left(m_{N} z_{-}\right)^{2} \rightarrow \tilde{\beta}$ asymptotically (6.31). We fix it to $\tilde{\beta}=17.65$. Finally, we fix the parameters of the emergent Fermi surface: $v_{F}=1$ (Fermi velocity), $\tilde{h}_{2}=1$ for simplicity, $\mu / m_{N}=$ 1.2 (chemical potential) for a typical nucleus

With these parameters fixed, we show in Figs. 5-7, the dense R-ratio versus $\mathrm{x}$ for different Fermi momenta $k_{F} / m_{N}=4,0.5,0.3$. For $k_{F} / m_{N} \geq 0.4 \approx k_{R} / m_{N}$ the contribution from the emergent $\mathrm{AdS}_{2}$ Fermi surface near the horizon is small but real. This contribution disappears for $k_{F}<k_{R}$ as we noted in (2.11), and only the $\mathrm{AdS}_{5}$ contribution far from the horizon remains. The contribution from the emergent $\mathrm{AdS}_{2}$ Fermi surface becomes comparable to the far horizon $\mathrm{AdS}_{5}$ contribution at large-x and only for large values of $k_{F} / m_{N}$. For all values of $k_{F} / m_{N}$ and most values of $x$, the leading black hole contribution is dominant. Some of the key features of DIS scattering on a nucleus are already captured by

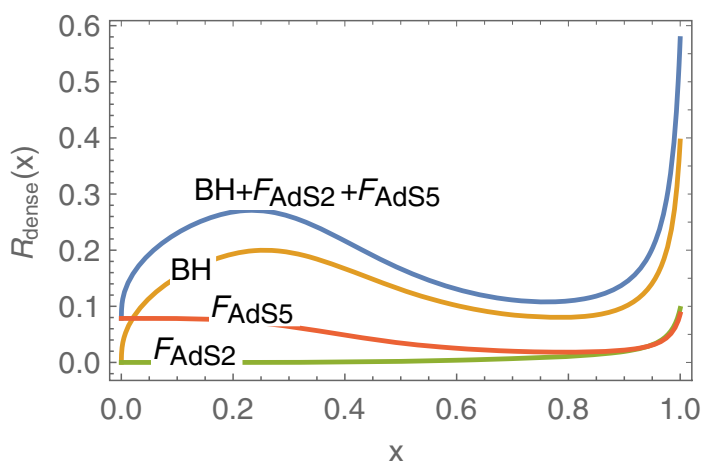

FIG. 5. Dense R-ratio (5.13) with $k_{F} / m_{N}=4$. See text.

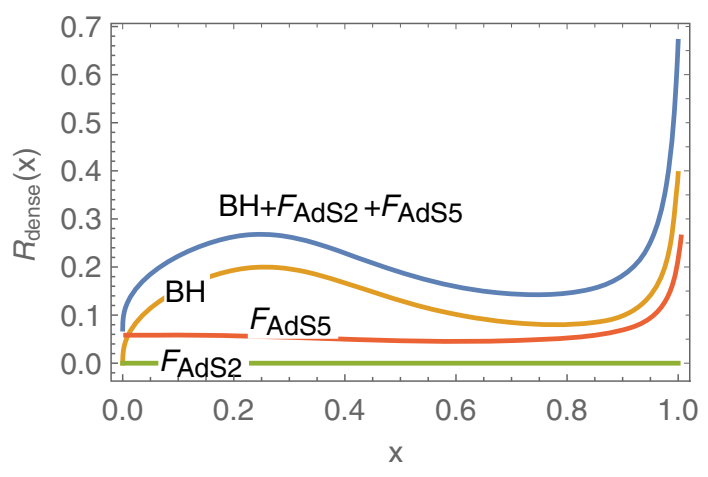

FIG. 6. Dense R-ratio (5.13) with $k_{F} / m_{N}=0.5$. See text. 


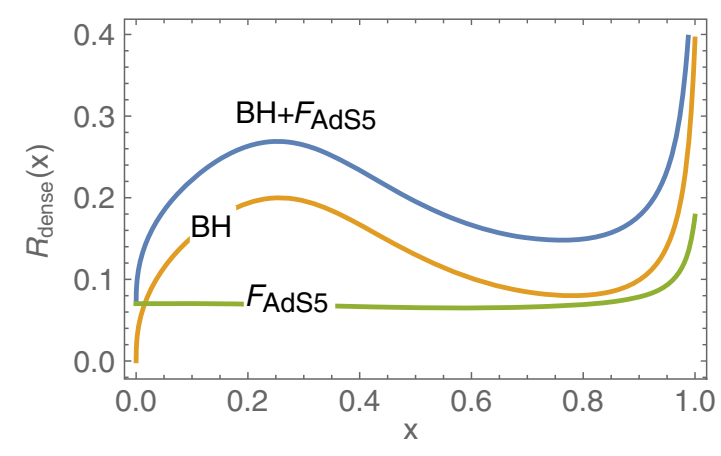

FIG. 7. Dense R-ratio (5.13) with $k_{F} / m_{N}=0.3$, and without the contribution from $\mathrm{AdS}_{2}$. See text.

Figs. 5-7 with shadowing and anti-shadowing at small-x due to the coherent scattering on the black hole, and Fermi motion at large-x that is increasingly apparent from DIS scattering on the $\mathrm{AdS}_{5}$ from the far horizon part at small $k_{F} / m_{N}$. We will return to these important physical issues below through a more realistic model for DIS scattering on a finite nucleus with comparison to the existing world-data from DIS scattering on light and heavy nuclei.

\section{DIS IN THE PROBE LIMIT: DILUTE REGIME}

We now consider scattering in the probe limit, where the bulk fermions carry a density without affecting the underlying $\mathrm{AdS}_{5}$ geometry (with or without a wall), i.e., $\frac{\mu}{\sqrt{\tilde{\alpha}}} \rightarrow 0$ with $\frac{\mu}{\sqrt{\tilde{\alpha}}} \times e_{R} \sqrt{\tilde{\alpha}}=\mu_{e}$ fixed where $\tilde{\alpha} \sim \frac{N_{c}}{N_{f}} \gg 1$ and $\mu$ is the chemical potential. This limit, can be regarded as the UV completion for the emergent Fermi surface in bulk, but also can be considered as DIS scattering on a very dilute nucleus. This amounts to using the free spectral form (3.16) with the substitution

$$
\operatorname{Im} \mathcal{G}_{F}^{\alpha \gamma}\left(\omega_{1}, k\right) \rightarrow \pi n_{F}\left(\omega_{1}, \vec{k}\right) \delta\left(k^{2}+\omega_{1}^{2}\right) \delta^{\alpha \gamma}
$$

Here $n_{F}\left(\omega_{1}, \vec{k}\right)=\theta\left(\mu_{e}-\left(k^{2}+\omega_{1}^{2}\right)^{\frac{1}{2}}\right)$ is the Fermi occupation factor for a fermion of momentum $\vec{k}$, mass $\omega_{1}$ and Fermi energy $\mu_{e}$, and the vacuum ( $\left.\mathrm{AdS}_{5}\right)$ wave functions. For the confining case, the mass $\omega_{1}$ is quantized. This analysis complements the one we have discussed recently using generic arguments based on a density expansion of a trapped Fermi liquid [15].

\section{A. Large-x}

With this in mind, consider the case of scattering in the ultraviolet region of the black hole, with the hard fermion of momentum $k+q$ and the remaining fermion of momentum $k$ treated in the probe approximation. This example will help clarify the relationship between our analysis and that in [21]. For that we use the vacuum propagator (3.15) for both the hard fermion and the density modified propagator (B6) and (6.1) for the soft fermion in (3.26),

$$
\begin{aligned}
\operatorname{Im} \tilde{G}_{F}^{\mu \nu}(q) & =\int \frac{d^{4} k}{(2 \pi)^{4}} \int d \omega_{1} \omega_{1} \int d \omega_{2} \omega_{2} \operatorname{Re} \operatorname{Tr}\left(\mathcal{G}_{F}\left(\omega_{1}, k+q\right) \Lambda^{\mu}\left(\omega_{1} ; q ; \omega_{2}\right) \mathcal{G}_{F}\left(\omega_{2}, k\right) \Lambda^{\nu}\left(\omega_{2} ; q ; \omega_{1}\right)\right), \\
& =\int \frac{d^{3} k}{(2 \pi)^{3}} \int_{-\left|q^{0}\right|}^{0} \frac{d k^{0}}{2 \pi} \int d \omega_{1} \omega_{1} \int d \omega_{2} \omega_{2} \operatorname{Tr}\left(\operatorname{Im} \mathcal{G}_{R}\left(\omega_{1}, k+q\right) \Lambda^{\mu}\left(\omega_{1} ; q ; \omega_{2}\right) \operatorname{Im} \mathcal{G}_{R}\left(\omega_{2}, k\right) \Lambda^{\nu}\left(\omega_{2} ; q ; \omega_{1}\right)\right)
\end{aligned}
$$

where the hard vertices are defined as

$$
\begin{aligned}
& \Lambda^{\mu}\left(\omega_{1} ; q ; \omega_{2}\right)=\int d r_{2} \sqrt{g\left(r_{2}\right)} \overline{\boldsymbol{\psi}}\left(r_{2}, \omega_{1}\right) Q^{\mu}\left(r_{2} ; q\right) \boldsymbol{\psi}\left(r_{2}, \omega_{2}\right), \\
& \Lambda^{\nu}\left(\omega_{2} ; q ; \omega_{1}\right)=\int d r_{1} \sqrt{g\left(r_{1}\right)} \overline{\boldsymbol{\psi}}\left(r_{1}, \omega_{2}\right) Q^{\nu}\left(r_{1} ; q\right) \boldsymbol{\psi}\left(r_{1}, \omega_{1}\right) .
\end{aligned}
$$

Here $\boldsymbol{\psi}(r, \omega)$ and $\overline{\boldsymbol{\psi}}(r, \omega)$ are the hard wave functions [21]

$$
\begin{aligned}
& \boldsymbol{\psi}(z, \omega)=z^{5 / 2}\left[J_{m R-1 / 2}(\omega z) P_{+}+J_{m R+1 / 2}(\omega z) P_{-}\right], \\
& \overline{\boldsymbol{\psi}}(z, \omega)=z^{5 / 2}\left[P_{-} J_{m R-1 / 2}(\omega z)+J_{m R+1 / 2}(\omega z) P_{+}\right],
\end{aligned}
$$

with the chiral projectors $P_{ \pm}=\frac{1}{2}\left(1 \pm \gamma_{5}\right)$. In this regime, the imaginary parts are reducible to on-shell delta functions

$$
\operatorname{Im}_{\mathcal{G}_{R}}\left(\omega_{1}, k+q\right) \operatorname{Im} \mathcal{G}_{R}\left(\omega_{2}, k^{0}, \vec{k}\right) \rightarrow\left(-(\not k+\not 1)+\omega_{1}\right) \pi \delta\left((k+q)^{2}+\omega_{1}^{2}\right)\left(-\not k+\omega_{2}\right) \pi n_{F}\left(\omega_{2}, \vec{k}\right) \delta\left(k^{2}+\omega_{2}^{2}\right)
$$


after using (6.1). Recall that $n_{F}(\omega, \vec{k})$ is the Fermi distribution for a fermion of mass $\omega$ and momentum $\vec{k}$ near the boundary in the probe limit. With this in mind, (6.2) becomes

$$
\begin{aligned}
\operatorname{Im} \tilde{G}_{F}^{\mu \nu}(q) \approx & \int \frac{d^{3} k}{(2 \pi)^{3}} \int_{-\left|q^{0}\right|}^{0} \frac{d k^{0}}{2 \pi} \int_{0}^{q^{2}} \frac{d \omega_{2}^{2}}{2} \int_{0}^{\left(q-\omega_{2}\right)^{2}} \frac{d \omega_{1}^{2}}{2} \\
& \times \operatorname{tr}\left(\left(-(\not k+\not q)+\omega_{1}\right) \Lambda^{\mu}\left(\omega_{1} ; q ; \omega_{2}\right)\left(-\not k+\omega_{2}\right) \Lambda^{\nu}\left(\omega_{2} ; q ; \omega_{1}\right)\right) \pi \delta\left((k+q)^{2}+\omega_{1}^{2}\right) \pi \delta\left(k^{2}+\omega_{2}^{2}\right), \\
= & \int \frac{d^{3} k}{(2 \pi)^{3}} \int_{-\left|q^{0}\right|}^{0} \frac{d k^{0}}{2 \pi} \int_{0}^{q^{2}} \frac{d \omega_{2}^{2}}{2} \int_{0}^{\left(q-\omega_{2}\right)^{2}} \frac{d \omega_{1}^{2}}{2} I_{z v}^{2}\left(\omega_{1}, \omega_{2}, q\right) \\
& \times \operatorname{tr}\left(\left(-(\not k+\not \mid)+\omega_{1}\right) \gamma^{\mu}\left(-\not k+\omega_{2}\right) \gamma^{\nu} P_{+}\right) \pi \delta\left((k+q)^{2}+\omega_{1}^{2}\right) \pi n_{F}\left(\omega_{2}, \vec{k}\right) \delta\left(k^{2}+\omega_{2}^{2}\right),
\end{aligned}
$$

with the physical condition $\omega_{1}+\omega_{2}<q$ (i.e., a meson or virtual photon of mass $q$ decaying into KK-fermions of masses $\omega_{1}$ and $\left.\omega_{2}\right)$, and

$$
\begin{aligned}
I_{z v}\left(\omega_{1}, \omega_{2}, q\right) & =e_{R} R^{4} q \int_{0}^{\infty} d z z^{2} J_{m R-1 / 2}\left(\omega_{1} z\right) J_{m R-1 / 2}\left(\omega_{2} z\right) K_{1}(q z) \\
& \approx e_{R} R^{4} \frac{2^{-(m R-1 / 2)}}{\Gamma(m R+1 / 2)} \omega_{2}^{m R-\frac{1}{2}} q \int_{0}^{\infty} d z z^{m R+\frac{3}{2}} J_{m R-1 / 2}\left(\omega_{1} z\right) K_{1}(q z) \\
& \approx 2 e_{R} R^{4}(m R+1 / 2) \frac{1}{q^{2}}\left(\frac{\omega_{2}}{q}\right)^{m R-\frac{1}{2}}\left(\frac{\omega_{1}}{q}\right)^{m R-\frac{1}{2}}\left(1+\frac{\omega_{1}^{2}}{q^{2}}\right)^{-\left(m R+\frac{3}{2}\right)}
\end{aligned}
$$

where we made use of the approximation

$$
J_{m R-1 / 2}\left(\omega_{2} z\right) \approx \frac{2^{-(m R-1 / 2)}}{\Gamma(m R+1 / 2)}\left(\omega_{2} z\right)^{m R-\frac{1}{2}}
$$

for $\omega_{2} z \ll 1$. Note that without making the approximation $\omega_{2} z \ll 1$, the above integral $I_{z \nu} \sim F_{4}\left(a, b ; c, d ;-\frac{\omega_{1}^{2}}{q^{2}},-\frac{\omega_{2}^{2}}{q^{2}}\right)$ $[25,26]$ where $F_{4}$ is the fourth Appell series of hypergeometric functions which is indeed convergent only for $\omega_{1}+\omega_{2}<q$.

The integral in (6.6) is in agreement with the R-current scattering on a dilatino in [21]. Evaluating the integral in (6.5) over $\omega_{1}$ using the delta-function $\delta\left(\omega_{1}^{2}-s_{k}\right)$, and using (6.6), we have

$\left.\operatorname{Im} \tilde{G}_{F}^{\mu \nu}(q) \approx \frac{\pi}{2} \int \frac{d^{3} k}{(2 \pi)^{3}} \int_{-\left|q^{0}\right|}^{0} \frac{d k^{0}}{2 \pi} \int_{0}^{q^{2}} \frac{d \omega_{2}^{2}}{2} I_{z v}^{2}\left(\sqrt{s_{k}}, \omega_{2}, q\right) \operatorname{Tr}\left((-\not k+\not q)+\sqrt{s_{k}}\right) \gamma^{\mu}\left(-\not k+\omega_{2}\right) \gamma^{\nu} P_{+}\right) \pi n_{F} \delta\left(k^{2}+\omega_{2}^{2}\right)$,

where $s_{k}=-(k+q)^{2}$. The evaluation of the remaining $k^{0}$-integral in (6.8) using the last delta-function, yields

$$
\operatorname{Im} \tilde{G}_{F}^{\mu \nu}(q) \approx \frac{\pi}{4} \int_{0}^{q^{2}} \frac{d \omega_{2}^{2}}{2} \int \frac{d^{3} k}{(2 \pi)^{3}} I_{z v}^{2}\left(\sqrt{s_{k}}, \omega_{2}, q\right) \operatorname{Tr}\left(\left(-(\not k+\not q)+\sqrt{s_{k}}\right) \gamma^{\mu}\left(-\not k+\omega_{2}\right) \gamma^{\nu} P_{+}\right) \frac{n_{F}\left(\omega_{2}, \vec{k}\right)}{2 E_{k}},
$$

where $E_{k}=\sqrt{|\vec{k}|^{2}+\omega_{2}^{2}}<\left|q^{0}\right|$, and $k^{0}=E_{k}$.

To extract the structure functions (3.2) from (6.9) we carry the spin trace by contacting with the timelike frame vector $n^{\mu}=(1,0,0,0)$,

$$
\begin{aligned}
n_{\mu} n_{\nu} \operatorname{Im} \tilde{G}_{F}^{\mu \nu}(q) & \approx \frac{\pi}{4} \int_{0}^{q^{2}} \frac{d \omega_{2}^{2}}{2} \int \frac{d^{3} k}{(2 \pi)^{3}} I_{z v}^{2}\left(\sqrt{s_{k}}, \omega_{2}, q\right) \operatorname{tr}\left(\left(-(\not k+\not q)+\sqrt{s_{k}}\right) \not h\left(-\not k+\omega_{2}\right) \not h P_{+}\right) \frac{n_{F}\left(\omega_{2}, \vec{k}\right)}{2 E_{k}}, \\
& \approx \pi \int_{0}^{q^{2}} \frac{d \omega_{2}^{2}}{2} \int \frac{d^{3} k}{(2 \pi)^{3}} I_{z v}^{2}\left(\sqrt{s_{k}}, \omega_{2}, q\right)\left((n \cdot k)^{2}-\frac{1}{2}\left(k \cdot q+\sqrt{s_{k}} \omega_{2}\right) n^{2}\right) \frac{n_{F}\left(\omega_{2}, \vec{k}\right)}{2 E_{k}},
\end{aligned}
$$

where we have assumed $n \cdot q \approx 0$ and $k^{2} \approx 0$. Note that the trace in (6.10) is the same trace evaluated in [21] for $\omega_{2}=0$ (see their Eq. 72). Using (3.2) with $x_{A}=-q^{2} / 2 P_{A} \cdot q$, we can now extract the structure functions $F_{1,2}\left(x_{A}, q^{2}\right)$ of a state with momentum $P_{A}^{\mu}$ from (6.10) 


$$
\begin{aligned}
& \tilde{F}_{2}\left(x_{A}, q^{2}\right) \approx 2 \pi^{2} \frac{q^{2}}{2 x_{A}\left(n \cdot P_{A}\right)^{2}} \int_{0}^{\Lambda^{2}<q^{2}} \frac{d \omega_{2}^{2}}{2} \int \frac{d^{3} k}{(2 \pi)^{3}} I_{z v}^{2}\left(\sqrt{s_{k}}, \omega_{2}, q\right)(n \cdot k)^{2} \frac{n_{F}\left(\omega_{2}, \vec{k}\right)}{2 E_{k}}, \\
& \tilde{F}_{1}\left(x_{A}, q^{2}\right) \approx-\pi^{2} \int_{0}^{\Lambda^{2}<q^{2}} \frac{d \omega_{2}^{2}}{2} \int \frac{d^{3} k}{(2 \pi)^{3}} I_{z v}^{2}\left(\sqrt{s_{k}}, \omega_{2}, q\right)\left(k \cdot q+\sqrt{s_{k}} \omega_{2}\right) \frac{n_{F}\left(\omega_{2}, \vec{k}\right)}{2 E_{k}} .
\end{aligned}
$$

We have $x_{k}=-q^{2} / 2 k \cdot q, \quad s_{k}=-(k+q)^{2} \approx-q^{2}\left(1-1 / x_{k}\right)$, and $k^{0}=E_{k}=\left(|\vec{k}|^{2}+\omega_{2}^{2}\right)^{\frac{1}{2}}<\left|q^{0}\right|$. We can reduce $I_{z v}\left(\sqrt{s_{k}}, \omega_{2}, q\right)$ in (6.6) in terms of $x_{k}$ as

$$
I_{z v}\left(\sqrt{s_{k}}, \omega_{2}, q\right) \approx 2 e_{R} R^{4}(m R+1 / 2) \omega_{2}^{m R-1 / 2} q^{-(m R+3 / 2)} x_{k}^{\frac{1}{2}(m R+7 / 2)}\left(1-x_{k}\right)^{\frac{1}{2}(m R-1 / 2)},
$$

for the mass range $\omega_{2} \leq \Lambda$. Using (6.12), we can rewrite the structure functions (6.11) in terms of $x_{k}$ as

$$
\begin{aligned}
& \tilde{F}_{2}\left(x_{A}, q^{2}\right) \approx 2^{2} \pi^{2} e_{R}^{2} R^{8}(\tau-1)^{2}\left(\frac{1}{q^{2}}\right)^{\tau-1} \frac{1}{x_{A}\left(n \cdot P_{A}\right)^{2}} \int_{0}^{\Lambda^{2}<q^{2}} \frac{d \omega_{2}^{2}}{2}\left(\omega_{2}^{2}\right)^{\tau-2} \int \frac{d^{3} k}{(2 \pi)^{3}} \frac{n_{F}\left(\omega_{2}, \vec{k}\right)}{2 E_{k}}(n \cdot k)^{2} x_{k}^{\tau+2}\left(1-x_{k}\right)^{\tau-2} \\
& \tilde{F}_{1}\left(x_{A}, q^{2}\right) \approx 2 \pi^{2} e_{R}^{2} R^{8}(\tau-1)^{2}\left(\frac{1}{q^{2}}\right)^{\tau-1} \int_{0}^{\Lambda^{2}<q^{2}} \frac{d \omega_{2}^{2}}{2}\left(\omega_{2}^{2}\right)^{\tau-2} \int \frac{d^{3} k}{(2 \pi)^{3}} \frac{n_{F}\left(\omega_{2}, \vec{k}\right)}{2 E_{k}} x_{k}^{\tau+1}\left(1-x_{k}\right)^{\tau-2},
\end{aligned}
$$

with the twist parameter is $\tau=m R+3 / 2$, following the approximation $\left(\omega_{2} \ll q\right)$

$$
\sqrt{s_{k}} \frac{\omega_{2}}{q^{2}} \approx\left(\frac{1}{x_{k}}-1\right)^{\frac{1}{2}} \frac{\omega_{2}}{q} \approx 0
$$

\section{B. Low-x}

In contrast to the dense limit in (4.13), the bulk-to-bulk graviton propagator in the probe limit, is given by

$$
G_{x y, x y}\left(\tilde{q}_{0}, \tilde{q}_{x}, z, z^{\prime}\right)=\int_{0}^{\infty} \frac{d \omega^{2}}{2} \frac{z^{2} J_{\Delta}(z \omega) z^{\prime 2} J_{\Delta}\left(z^{\prime} \omega\right)}{-t+\omega^{2}-i \epsilon}
$$

where $t=-\tilde{q}^{2}=\tilde{q}_{0}^{2}-\tilde{q}_{x}^{2}$. Therefore, $G_{x y, x y}\left(\tilde{q}_{0}=0, \tilde{q}_{x}=0, z, z^{\prime}\right) \neq 0$ does not vanish in the probe limit. In this limit, the graviton exchange Reggeizes by including higher spin-j (stringy) exchange as

$$
\mathcal{K}\left(j, \tilde{q}_{0}, \tilde{q}_{x}, z, z^{\prime}\right)=\int_{0}^{\infty} \frac{d \omega^{2}}{2} \frac{z^{2} J_{\tilde{\Delta}(j)}(z \omega) z^{\prime 2} J_{\tilde{\Delta}(j)}\left(z^{\prime} \omega\right)}{-t+\omega^{2}-i \epsilon}
$$

with $\tilde{\Delta}(j)=(4+2 \sqrt{\lambda}(j-2))^{\frac{1}{2}}$.

With this in mind, we now consider the case of the one-loop fermionic contribution in the probe limit at low-x. In this regime, the bulk-to-bulk fermion propagator is of the form [20]

$$
D_{F}\left(r, r^{\prime}, k\right)=-\int \frac{d \omega^{2}}{2} \boldsymbol{\psi}(r) \frac{-i \not k+\omega}{k^{2}+\omega^{2}-i \epsilon} \overline{\boldsymbol{\psi}}\left(r^{\prime}\right) .
$$

Note that only in this section, we have added an extra factor of $i$ in the gamma matrix in comparison to (3.18) and replaced $\omega$ by $-\omega$ to make the comparison with standard results easier. Inserting (4.11) in (4.8), we obtain the on-shell one-loop effective action $\mathcal{S}_{F}\left[A_{\mu}^{(0)} \equiv n_{\mu}\right]=n_{\mu} n_{\nu} \operatorname{Im} \tilde{G}_{F}^{\mu \nu}(q)$, 


$$
\begin{aligned}
n_{\mu} n_{\nu} \operatorname{Im} \tilde{G}_{F}^{\mu \nu}(q) \approx & \int \frac{d^{3} k}{(2 \pi)^{3}} \int_{-\left|q_{0}\right|}^{0} \frac{d k^{0}}{2 \pi} \int d r \sqrt{-g}\left(n_{\mu} n_{\nu} \frac{q^{4} R^{6}}{r^{4}}\left[K_{0}^{2}\left(q R^{2} / r\right)+K_{1}^{2}\left(q R^{2} / r\right)\right]+q_{\mu} q_{\nu} n^{2} \frac{q^{2} R^{6}}{r^{4}} K_{1}^{2}\left(q R^{2} / r\right)\right) \\
& \times\left.\operatorname{Tr}\left(\operatorname{Im} D_{R}(r, r, k) \Gamma^{\mu}(-i k)_{\alpha} g^{\alpha \nu}\right) \operatorname{Im} \mathcal{V}_{R}\right|_{t=0} \\
\approx & C_{\lambda} \int d r \sqrt{-g} \sqrt{g^{i i}} g^{i i} \int_{0}^{q^{2}} \frac{d \omega^{2}}{2} \int \frac{d^{3} k}{(2 \pi)^{3}} \frac{n_{F}(\omega, \vec{k})}{2 E_{k}} \\
& \times\left((n \cdot k)^{2} \frac{q^{4} R^{6}}{r^{4}}\left[K_{0}^{2}\left(q R^{2} / r\right)+K_{1}^{2}\left(q R^{2} / r\right)\right]+\frac{q^{4}}{4 x^{2}} n^{2} \frac{q^{2} R^{6}}{r^{4}} K_{1}^{2}\left(q R^{2} / r\right)\right) \\
& \times\left(\omega R^{2} / r\right)^{2 m R-1}\left(R^{2} / r\right)^{5} \sum_{j=1}^{\infty} \frac{2 \sqrt{4 \pi \lambda}}{s}\left(r / R^{2}\right)^{3} \delta\left(r-r_{j}\right) \\
& \approx C_{\lambda}\left(\frac{1}{q^{2}}\right)^{\tau-1} \int_{0}^{q^{2}} \frac{d \omega^{2}}{2}\left(\omega^{2}\right)^{\tau-2} \int \frac{d^{3} k}{(2 \pi)^{3}} \frac{n_{F}(\omega, \vec{k})}{2 E_{k}} \sum_{j=1}^{\infty} \frac{w_{j}}{2 j} w_{j}^{2 \tau+3}\left(\frac{(n \cdot k)^{2}}{q^{2}}\left[K_{0}^{2}\left(w_{j}\right)+K_{1}^{2}\left(w_{j}\right)\right]+\frac{1}{4 x_{k}^{2}} n^{2} K_{1}^{2}\left(w_{j}\right)\right) \\
& \approx C_{\lambda}\left(\frac{1}{q^{2}}\right)^{\tau-1} \int_{0}^{q^{2}} \frac{d \omega^{2}}{2}\left(\omega^{2}\right)^{\tau-2} \int \frac{d^{3} k}{(2 \pi)^{3}} \frac{n_{F}(\omega, \vec{k})}{2 E_{k}} \int_{0}^{\infty} d w w^{2 \tau+3}\left(\frac{(n \cdot k)^{2}}{q^{2}}\left[K_{0}^{2}(w)+K_{1}^{2}(w)\right]+\frac{1}{4 x_{k}^{2}} n^{2} K_{1}^{2}(w)\right) \\
& \approx C_{\lambda}\left(\frac{1}{q^{2}}\right)^{\tau-1} \int_{0}^{q^{2}} \frac{d \omega^{2}}{2}\left(\omega^{2}\right)^{\tau-2} \int \frac{d^{3} k}{(2 \pi)^{3}} \frac{n_{F}(\omega, \vec{k})}{2 E_{k}}\left(\frac{(n \cdot k)^{2}}{q^{2}}\left[I_{0,2 \tau+3}+I_{1,2 \tau+3}\right]+\frac{1}{4 x_{k}^{2}} n^{2} I_{1,2 \tau+3}\right) .
\end{aligned}
$$

Here $E_{k}=\left(|\vec{k}|^{2}+\omega^{2}\right)^{\frac{1}{2}}<\left|q^{0}\right|, k^{0}=E_{k}$, and $x_{k}=-\frac{q^{2}}{2 k \cdot q}$. Also we have set $r_{j}=R \sqrt{\alpha^{\prime} s} / 2 \sqrt{j}, w_{j}=q R^{2} / r_{j}=q z_{j}$,

$$
C_{\lambda}=\frac{\pi c_{5} R^{4}}{2 \sqrt{4 \pi \lambda}} \frac{2^{-(2 m R-1)}}{\Gamma^{2}(m R+1 / 2)}
$$

and defined the integrals

$$
I_{j, n}=\int_{0}^{\infty} d w w^{n} K_{j}^{2}(w)=2^{n-2} \frac{\Gamma(\nu+j) \Gamma(\nu-j) \Gamma(\nu)^{2}}{\Gamma(2 \nu)}
$$

with $\nu=\frac{1}{2}(n+1)$. They are related to each other recursively $(n-1) I_{1, n}=(n+1) I_{0, n}$.

The structure functions of the nuclei at small-x in the probe limit are given by

$$
\begin{aligned}
& \tilde{F}_{2}\left(x_{A}, q^{2}\right) \approx 2 \pi C_{\lambda}\left(\frac{1}{q^{2}}\right)^{\tau-1} \frac{1}{2 x_{A}\left(n \cdot P_{A}\right)^{2}} \int_{0}^{\Lambda^{2}<q^{2}} \frac{d \omega^{2}}{2}\left(\omega^{2}\right)^{\tau-2} \int \frac{d^{3} k}{(2 \pi)^{3}} \frac{n_{F}(\omega, \vec{k})}{2 E_{k}}(n \cdot k)^{2}\left[I_{0,2 \tau+3}+I_{1,2 \tau+3}\right] \\
& \tilde{F}_{1}\left(x_{A}, q^{2}\right) \approx 2 \pi C_{\lambda}\left(\frac{1}{q^{2}}\right)^{\tau-1} \int_{0}^{\Lambda^{2}<q^{2}} \frac{d \omega^{2}}{2}\left(\omega^{2}\right)^{\tau-2} \int \frac{d^{3} k}{(2 \pi)^{3}} \frac{n_{F}(\omega, \vec{k})}{2 E_{k}}\left(\frac{1}{4 x_{k}^{2}} I_{1,2 \tau+3}\right) .
\end{aligned}
$$

The effects caused by the diffusion in the radial direction on the structure functions far from the black hole at low-x, are discussed in Appendix E.

\section{Normalized structure functions}

To normalize the structure functions in the probe limit, we recall that the bulk density and bulk energy density follows from the holographic principle as

$$
\begin{aligned}
& \tilde{n}(q z \ll 1)=\int_{0}^{\Lambda^{2}} \frac{d \omega^{2}}{2} I_{K}(q z \ll 1, \omega) \int_{0}^{k_{F}(\omega)} \frac{d^{3} k}{(2 \pi)^{3}} \\
& \tilde{\epsilon}(q z \ll 1)=\int_{0}^{\Lambda^{2}} \frac{d \omega^{2}}{2} I_{K}(q z \ll 1, \omega) \int_{0}^{k_{F}(\omega)} \frac{d^{3} k}{(2 \pi)^{3}} \sqrt{k^{2}+\omega^{2}}
\end{aligned}
$$

where $k_{F}(\omega)=\sqrt{\mu_{e}^{2}-\omega^{2}}$, and 


$$
\begin{aligned}
I_{K}(q z \ll 1, \omega) & =R^{4} \int_{0}^{z_{-}} d z z^{2 \tau-3}\left(\omega^{2}\right)^{\tau-2} \\
& =\frac{1}{2} \frac{R^{4}}{\tau-1}\left(\omega^{2} z_{-}^{2}\right)^{\tau-1} \omega^{-2}
\end{aligned}
$$

after taking $q z K_{1}(q z \ll 1) \approx 1$. The $\omega$-integration in (6.22) is carried over the bulk spectral density $I_{K}(0, \omega)$ with an upper cutoff $\Lambda$. In the conformal case, the cutoff is a priori arbitrary. In the conformally broken case, say a hard wall at $z=z_{-}$, we can set $z_{-} \Lambda=z_{-} m_{N}$ in (6.22) and assuming $\Lambda$ large, to pick only the nucleon ground state. A higher cutoff would include higher excited states of the nucleon. With this in mind, we can first undo the k-integration by approximating it near the Fermi surface, and then undo the $\omega$-integration by keeping only the leading contribution for $\frac{1}{\beta} \equiv\left(z_{-} m_{N}\right)^{2}>1$,

$$
\begin{aligned}
& \tilde{n}(q z \ll 1) \approx \frac{1}{8 \pi^{2}} \frac{R^{4}}{(\tau-1) \beta^{\tau-1}} k_{F}^{3} \\
& \tilde{\epsilon}(q z \ll 1) \approx \frac{1}{8 \pi^{2}} \frac{R^{4}}{(\tau-1) \beta^{\tau-1}} k_{F}^{3} E_{F}
\end{aligned}
$$

with $E_{F}=\left(k_{F}^{2}+m_{N}^{2}\right)^{\frac{1}{2}}$. We now identify the bulk density $\tilde{n}=A / V_{A}$ as the density of a fixed target say a nucleus, with A-nucleons in a fixed volume $V_{A}$ and a total energy $E_{A}=A E_{F}$. The normalized structure $F_{1,2}$ are then related to our earlier and un-normalized structure functions $\tilde{F}_{1,2}$ through

$$
F_{1,2} \equiv 2 E_{A} V_{A} \tilde{F}_{1,2}=2 A E_{A} \frac{\tilde{F}_{1,2}}{\tilde{n}} .
$$

\section{Case-1 (Large-x)}

The normalized structure functions at large-x follow by inserting (6.21) and (6.24) into (6.25) with $\beta=1 /\left(m_{N} z_{-}\right)^{2}$

$$
\begin{aligned}
& \frac{F_{2}\left(x, q^{2}\right)}{A} \approx 8 \pi^{2}(\tau-1)^{2} e_{R}^{2}\left(\frac{\beta m_{N}^{2}}{q^{2}}\right)^{\tau-1} x_{F}^{\tau+1}\left(1-x_{F}\right)^{\tau-2} \\
& \frac{F_{1}\left(x, q^{2}\right)}{A} \approx 4 \pi^{2}(\tau-1)^{2} e_{R}^{2}\left(\frac{\beta m_{N}^{2}}{q^{2}}\right)^{\tau-1} A x_{F}^{\tau+1}\left(1-x_{F}\right)^{\tau-2} .
\end{aligned}
$$

We define the $\mathrm{x}$-fractions

$$
\begin{aligned}
& x_{F}=\frac{q^{2}}{-2 p_{F} \cdot q} \approx \frac{q^{2}}{2 E_{F} \omega}=\frac{x m_{N}}{E_{F}} \\
& x_{A}=\frac{q^{2}}{-2 P_{A} \cdot q} \approx \frac{-q^{2}}{2 E_{A} \omega}=\frac{x_{F}}{A}
\end{aligned}
$$

and note that the large-x structure functions in (6.26) in the probe approximation obey the analogue of the Callan-Gross relation $F_{2}=2 x_{A} F_{1}$ for a holographic and dilute nucleus. Also we note that (6.26) are analogous to the so-called structure functions of the nucleus obtained through the so-called $\mathrm{x}$-scaling of the structure functions of the nucleon.

\section{Case-2 (Small-x):}

Doing the momentum integrals in (6.21) near $k \rightarrow k_{F}$ and doing the appropriate normalization as in the large- $x$ regime, we find

$$
\begin{aligned}
& \frac{F_{2}\left(x, q^{2}\right)}{A} \approx \pi C_{\lambda}\left(\frac{\beta m_{N}^{2}}{q^{2}}\right)^{\tau-1} \frac{1}{x_{F}}\left[I_{0,2 \tau+3}+I_{1,2 \tau+3}\right] \\
& \frac{F_{1}\left(x, q^{2}\right)}{A} \approx \pi C_{\lambda}\left(\frac{\beta m_{N}^{2}}{q^{2}}\right)^{\tau-1} A\left(\frac{1}{4 x_{F}^{2}} I_{1,2 \tau+3}\right),
\end{aligned}
$$

\section{R-ratio in the probe limit}

We define the R-ratio of the nucleus in the probe (dilute) limit as

$$
R_{\text {dilute }}\left(x, q^{2}\right) \equiv \frac{\frac{1}{A} F_{2}^{\text {dilute }}\left(x, q^{2}\right)}{F_{2}^{\text {nucleon }}\left(x, q^{2}\right)}
$$

where $F_{2}^{\text {dilute }}\left(x, q^{2}\right)$ is given by the sum of (6.26) for large-x and (6.28) for small-x,

$$
\begin{aligned}
\frac{F_{2}^{\text {dilute }}\left(x, q^{2}\right)}{A} \approx & f\left(\frac{\beta m_{N}^{2}}{q^{2}}\right)\left(\frac{\beta m_{N}^{2}}{q^{2}}\right)^{\tau-1} \\
& \times\left(8 \pi^{2}(\tau-1)^{2} e_{R}^{2} x_{F}^{\tau+1}\left(1-x_{F}\right)^{\tau-2}\right. \\
& \left.+\frac{c_{5} \pi^{2}}{2 \sqrt{4 \pi \lambda}} \frac{\left[I_{0,2 \tau+3}+I_{1,2 \tau+3}\right]}{2^{(2 \tau-4)} \Gamma^{2}(\tau-1)} \frac{1}{x_{F}^{j}}\right),
\end{aligned}
$$

and $F_{2}^{\text {nucleon }}\left(x, q^{2}\right)$ is given by

$$
\begin{aligned}
\frac{F_{2}^{\text {nucleon }}\left(x, q^{2}\right)}{A}= & f\left(\frac{\beta m_{N}^{2}}{q^{2}}\right)\left(\frac{\beta m_{N}^{2}}{q^{2}}\right)^{\tau-1} \\
& \times\left(8 \pi^{2}(\tau-1)^{2} e_{R}^{2} x^{\tau+1}(1-x)^{\tau-2}\right. \\
& \left.+\frac{c_{5} \pi^{2}}{2 \sqrt{4 \pi \lambda}} \frac{\left[I_{0,2 \tau+3}+I_{1,2 \tau+3}\right]}{2^{(2 \tau-4)} \Gamma^{2}(\tau-1)} \frac{1}{x^{j}}\right) .
\end{aligned}
$$

The additional multiplicative function $f(l)$ with argument $\imath=\beta m_{N}^{2} / q^{2}$ asymptotes a constant in the DIS regime which is our $\mathrm{AdS}_{5}$ result. The specific form of $f(\iota)$ depends on the details of the confining model for finite $q^{2}$ (see for example Eq. (3.28) and Eq. (4.3) in [11] for the soft-wall model) but in the DIS limit, the asymptotic constant can be reabsorbed through a shift $\beta \rightarrow \tilde{\beta}$. We recall that $\beta m_{N}^{2}=$ $1 / z_{-}^{2}$ is related to the confining scale here, and that $x_{F} E_{F}=x m_{N}$. Also we have in (6.28) $j=1$ in the absence of transverse diffusion or curvature corrections. When the latter are included $j \rightarrow 1-\mathcal{O}(1 / \sqrt{\lambda})$. The structure 


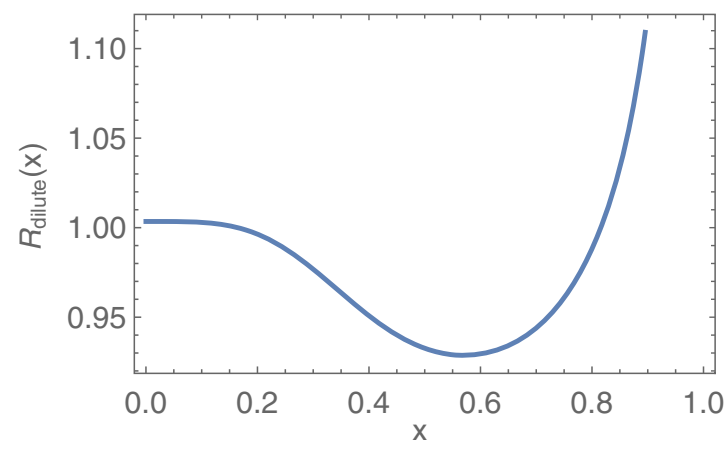

FIG. 8. Dilute R-ratio (6.29) for $k_{F} / m_{N}=0.3$. See text.

function of the proton follows from (6.30) by setting $k_{F}=$ 0 or through the substitution $x_{F} \rightarrow x$. The R-ratio for the probe or dilute limit is independent of $\beta \rightarrow \tilde{\beta}$ and $q^{2}$. Note that the first contribution is proportional to $e_{R}^{2} \lambda^{0}$ while the second is proportional to $e_{R}^{0} / \sqrt{\lambda}$ independent of the R-charge, as expected for Pomeron-like exchange.

In Fig. 8 we show the behavior of the dilute R-ratio (6.29) versus $x$ for fixed Fermi momentum $k_{F} / m_{N}=0.3$. The holographic parameters used are fewer but consistent with those used for the dense R-ratio in Fig. 5. Specifically, we have used: $e_{R}=0.3$ (R-charge of the bulk fermion), $2 \pi^{2} c_{5} / \sqrt{4 \pi \lambda}=0.01$ (strong coupling), $\tau=3$ (hard scaling exponent), $j=0.08$ (Pomeron intercept). In the dilute case, the R-ratio is dominant at large- $\mathrm{x}$ and asymptotes 1 at small$\mathrm{x}$. Clearly visible is the EMC-like effect for $0.2<x<0.8$ and the Fermi motion for $x>0.8$. We have checked that the overall features of Fig. 8 remain unchanged for smaller values of $e_{R}$ but fixed $k_{F} / m_{N}$ in conformity with the probe limit. This holographic behavior is very similar to the one we presented recently using general arguments [15].

\section{HYBRID MODEL FOR DIS SCATTERING ON A FINITE NUCLEUS}

Nuclei to a large extent are a collection of nucleons trapped by a mean-field usually the result of a Hartree-Fock approximation to the two-body and dominant interaction. Three- and higher-body interactions are suppressed as expected from the saturation properties and the bulk compressibility of nuclear matter.

DIS scattering on a nucleus is expected to be dominated by incoherent scattering on a dilute collection of nucleons at intermediate- and large- $x$, with modifications at small-x due coherent scattering induced by the residual and small two- and three-body mediated interactions following the rapid growth in parton- $x$.

A way to capture this, short of a more systematic density expansion outlined in [15], is to suggest that the nucleus structure function $F_{2}^{\text {nucleus }}\left(x, q^{2}\right)$ is composed of the dilute contribution (6.30) warped by $\mathrm{AdS}_{5}$ (low density and dominant contribution) plus the dense and quantum corrected black hole (5.14) (high density and subdominant contribution) with a new mixing coefficient $\mathbb{C}_{2}$ to be determined from the best fit to the world-data. In principle $\mathbb{C}_{2}$ can be obtained through a reorganization of the present scattering analysis, but we currently do not know how to achieve it. With this in mind, we define

$$
\frac{F_{2}^{\text {nucleus }}\left(x, q^{2}\right)}{A}=\frac{F_{2}^{\text {dense }}\left(x, q^{2}\right)}{A}+\mathbb{C}_{2} \frac{F_{2}^{\text {dilute }}\left(x, q^{2}\right)}{A}
$$

and the corresponding nucleus R-ratio as

$$
R_{\text {nucleus }}\left(x, q^{2}\right) \equiv \frac{\frac{1}{A} F_{2}^{\text {nucleus }}\left(x, q^{2}\right)}{F_{2}^{\text {nucleon }}\left(x, q^{2}\right)} .
$$

To compare the holographic results following from (7.2) to the world-data from DIS scattering on heavy and light nuclei, all the holographic parameters are set as before: $\tilde{\alpha}=N_{c} / 4 N_{f}=1$ (ratio of branes), $2 \pi^{2} c_{5} / \sqrt{4 \pi \lambda}=0.01$ (strong coupling) and $e_{R}=0.3$ (charge of the probe fermions), $\tau=3$ (hard scaling law), $j=0.08$ (Pomeron intercept) and $\beta \rightarrow \tilde{\beta}=17.65$ (confining scale). The parameters of the emergent Fermi surface are also fixed as before: $v_{F}=1$ (Fermi velocity), $\tilde{h}_{2}=1$ for simplicity,

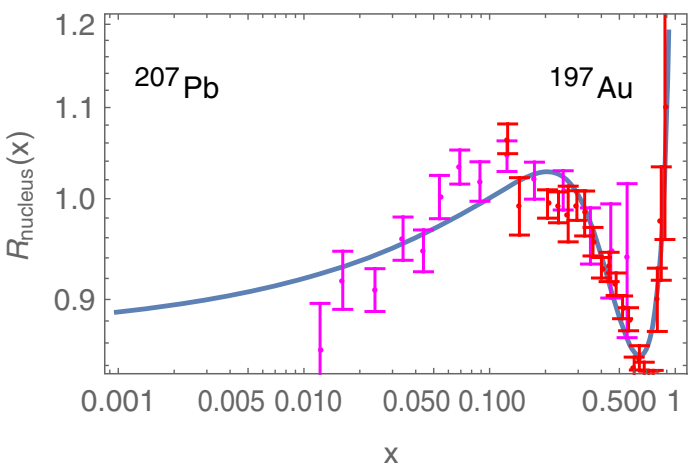

(b)

FIG. 9. Nucleus R-ratio (7.2) for Lead Pb with $\mathrm{A}=207$ and Gold $\mathrm{Au}$ with $\mathrm{A}=197$. We have fixed $k_{F} / m_{N}=0.395$. See text. The data points are from [29,30] for $\mathrm{Pb}$ (pink), and [31] for $\mathrm{Au}$ (red), see also Fig. 7 in [32]. 
$\mu / m_{N}=1.2$ (chemical potential for a typical nucleus), and $k_{F} / m_{N}=0.395$ (for $\mathrm{Au}, \mathrm{Pb}$, and $\mathrm{Fe}$ ), 0.277 (for C), 0.119 (for He) (Fermi momentum). We note that our choices for the nuclei Fermi momenta are very close to those extracted from quasielastic electron scattering experiments on nuclei [27] modeled using the Fermi gas model [28]. The parameter $\mathbb{C}_{1}=0.07$ fixes the quantum correction to the black hole due to the far horizon contribution. Our analysis of the data shows that the mixing parameter for the best fit is $\mathbb{C}_{2}=0.793$. Clearly, there is some form of double counting of the quantum corrections at large-x, but this is minimal since $\mathbb{C}_{1} / \mathbb{C}_{2}=0.09$.

We show in Figs. 9-12(a), the nucleus R-ratio versus $x$ for $\mathrm{Au}, \mathrm{Pb}, \mathrm{Fe}, \mathrm{C}$, and $\mathrm{He}$ on a linear scale. The overall

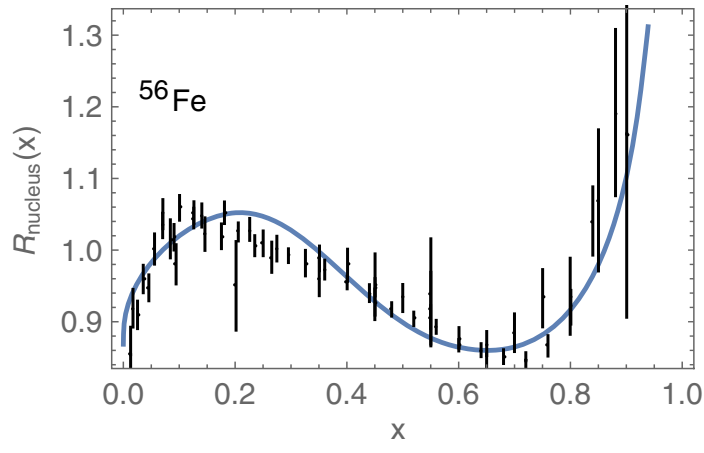

(a)

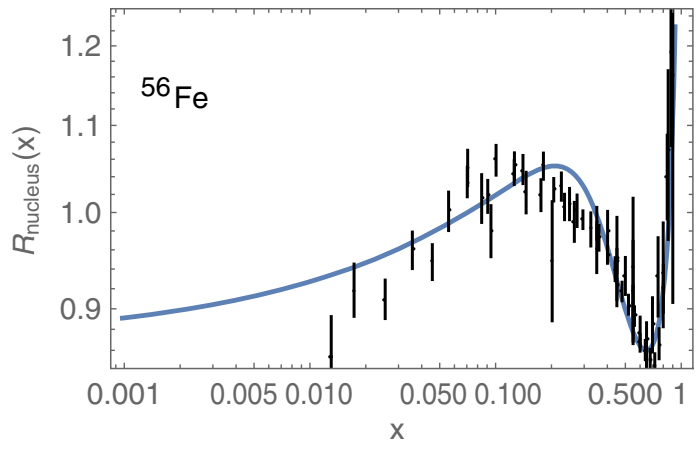

(b)

FIG. 10. Nucleus R-ratio (7.2) for Iron Fe with $\mathrm{A}=56$. We have fixed $k_{F} / m_{N}=0.395$. See text. The data points are from [29,30,33,34], see also Fig. 5 in [32].

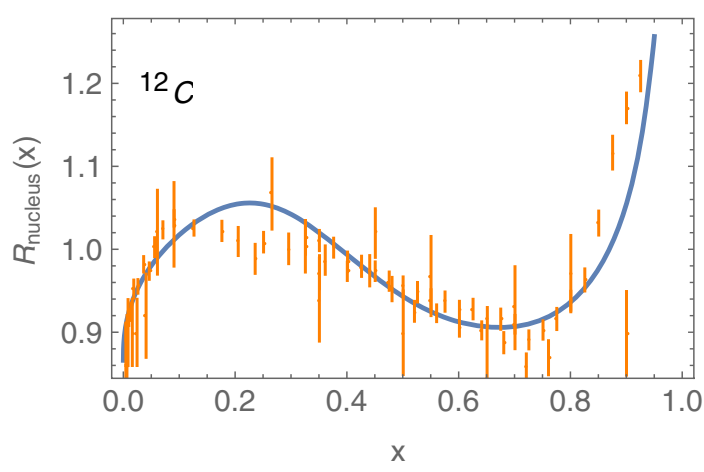

(a)

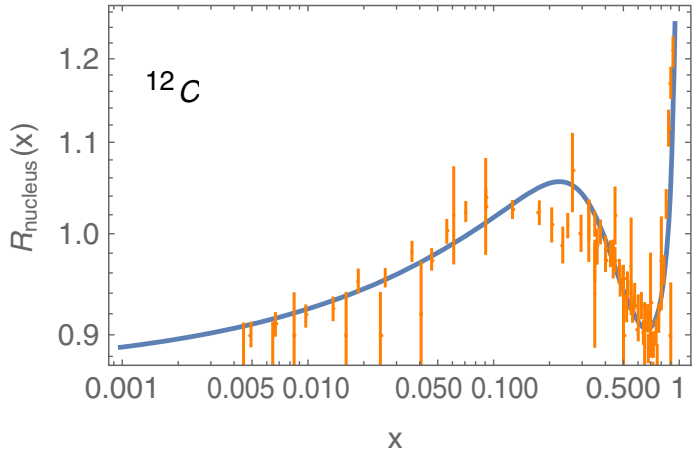

(b)

FIG. 11. Nucleus R-ratio (7.2) for Carbon $\mathrm{C}$ with $\mathrm{A}=12$. We have fixed $k_{F} / m_{N}=0.277$. See text. The data points are from [31,35-37], see also Fig. 3 in [32].

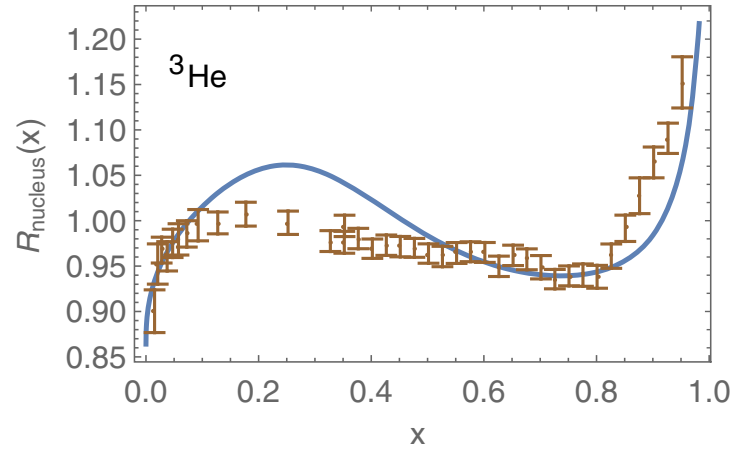

(a)

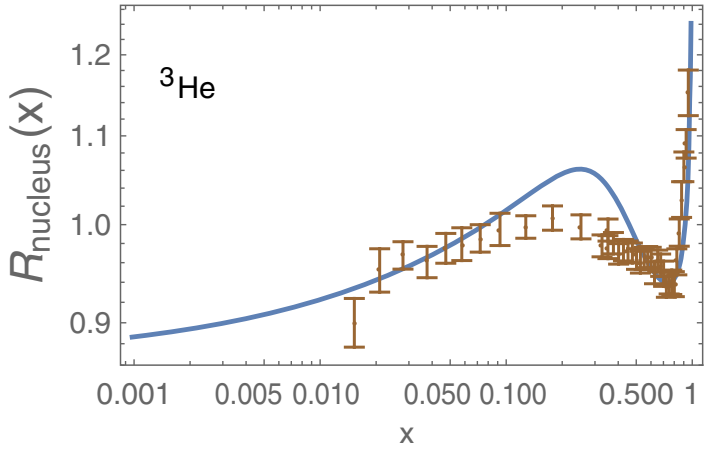

(b)

FIG. 12. Nucleus R-ratio (7.2) for Helium He with $\mathrm{A}=3$. We have fixed $k_{F} / m_{N}=0.119$. See text. The data points are from [35,38], see also Fig. 1 in [32]. 
agreement with the heavy and heavy-light nuclei data is fair throughout, the exception being He where the holographic model overshoots in the antishadowing region. This may be an indication that the coherent scattering captured by the black hole should be weaker, which is sensible. Figs. 9-12(b) display the same ratio on a semilogarithmic scale to highlight the low-x contribution where the data are scarce. A key proposal of the future Electron-Ion-Collider (EIC) is to provide measurements for the nuclear R-ratio in this region.

Remarkably, the R-ratio (7.2) exhibits shadowing for $x<0.1$, antishadowing for $0.1<x<0.3$, the EMC-like effect for $0.3<x<0.8$ and Fermi motion for $x>0.8$. As we noted earlier, shadowing and antishadowing are caused by coherent DIS scattering on the dense component in (7.1) due to the underlying black hole in our analysis, while the EMC effect and Fermi motion are mostly due to incoherent DIS scattering on the dilute component in (7.1) with the nucleus mostly composed of individual nucleons warped by $\mathrm{AdS}_{5}$.

\section{CONCLUSIONS}

In the double limit of a large number of colors and strong coupling, DIS scattering off an extremal black hole is of order $N_{c}^{2}$ following from the absorption of the bulk $\mathrm{R}$-current by the black hole. The corresponding structure functions are dominated by low-x. Scattering off the black hole is the ultimate coherent scattering off a dense nucleus with strong shadowing as we noted in [13].

To order $N_{c}^{0}$, DIS scattering is off holographic fermions hovering near the black-hole horizon due to quantum pair creation, and warped holographic fermions far from the black hole horizon near the boundary. Close to the horizon, the geometry is that of $\mathrm{AdS}_{2} \times \mathrm{R}^{3}$ with an emergent Fermi surface and anomalous scaling laws. DIS scattering off these bulk fermions show that their structure functions on the boundary exhibit anomalous exponents and modified hard scattering rules in comparison to scattering off bulk fermions in the dilute or probe limit. DIS scattering off these fermions exhibit Fermi motion at large-x.

DIS scattering on a wide range of nuclei maybe captured by a hybrid holographic model whereby most of the DIS scattering is incoherent and off a holographic Fermi liquid warped by $\mathrm{AdS}_{5}$ at intermediate- and large-x, with the remainder following from coherent scattering off a quantum corrected RN-AdS black hole at small-x. The ensuing results agree remarkably with the existing data on DIS scattering on finite nuclei over a broad range of parton-x. More data at low-x from the planned EIC collider will be welcome for a better understanding of the coherent scattering through the black-hole mechanism suggested here.

\section{ACKNOWLEDGMENTS}

This work was supported by the U.S. Department of Energy under Contract No. DE-FG-88ER40388.

\section{APPENDIX A: CONVENTIONS IN CURVED SPACE}

The gamma matrices in curved and tangent space used to analyze the Dirac equation in the extremal RN-AdS black hole will be made explicit here. For that, consider the generic line element in curved space

$$
d s^{2}=-g_{t t} d t^{2}+g_{r r} d r^{2}+g_{i i} d x_{i}^{2}
$$

If we refer to the indices in curved space by $\mu, \nu$ (also $t, i$ ) and those in the tangent space by $a, b$ (also $\underline{t}, \underline{i}$ ) then the gamma matrices are related by

$$
\Gamma^{\mu}=\Gamma^{a} e_{a}^{\mu}, \quad \Gamma^{r}=\sqrt{g^{r r}} \Gamma^{\underline{r} .}
$$

If we set the vierbeins as [39]

$$
e^{\underline{t}}=\sqrt{g_{t t}} d t, \quad e^{-\underline{i}}=\sqrt{g_{i i}} d x^{i},
$$

then we have

$\Gamma^{t}=\sqrt{g^{t t}} \Gamma^{\underline{t}}, \quad \Gamma^{i}=\sqrt{g^{i i}} \Gamma^{\underline{i}}, \quad \Gamma^{r}=\sqrt{g^{r r}} \Gamma^{\underline{r}}$.

In the tangent space, the gamma matrices read [19]

$$
\begin{array}{ll}
\Gamma^{\underline{t}}=\left(\begin{array}{cc}
i \sigma_{1} & 0 \\
0 & i \sigma_{1}
\end{array}\right) & \Gamma^{\underline{r}}=\left(\begin{array}{cc}
\sigma_{3} & 0 \\
0 & \sigma_{3}
\end{array}\right) \\
\Gamma \underline{x}=\left(\begin{array}{cc}
-\sigma_{2} & 0 \\
0 & \sigma_{2}
\end{array}\right) & \Gamma^{\underline{y}}=\left(\begin{array}{cc}
0 & -\sigma_{2} \\
-\sigma_{2} & 0
\end{array}\right) \\
\Gamma^{\underline{z}}=\left(\begin{array}{cc}
0 & i \sigma_{2} \\
-i \sigma_{2} & 0
\end{array}\right) . &
\end{array}
$$

The nonvanishing spin connections are

$$
\omega_{\underline{t r}}=-f_{0} e^{\underline{t}}, \quad \omega_{\underline{i r}}=f_{1} e^{\underline{i}}
$$

with

$$
f_{0} \equiv \frac{1}{2} \frac{g_{t t}^{\prime}}{\tilde{g}_{t t}} \sqrt{g^{r r}}, \quad f_{1} \equiv \frac{1}{2} \frac{g_{i i}^{\prime}}{g_{i i}} \sqrt{g^{r r}}
$$

\section{APPENDIX B: SOFT SPINORS}

The soft normalizable wave functions were constructed in [39], we reproduce them here for completeness. The Dirac equation in the $\mathrm{AdS}_{2} \times \mathrm{R}^{3}$ geometry is solved by the rescaled spinors

$\boldsymbol{\psi}_{1}(r ; \vec{k})=\left(-g g^{r r}\right)^{-\frac{1}{4}}\left(\begin{array}{c}\boldsymbol{\Phi}_{1} \\ 0 \\ 0\end{array}\right) \times \sqrt{\frac{r_{-}}{R^{2}}} \times\left(\frac{r_{-}}{R^{2}}\right)^{\nu_{k}}$,

$\boldsymbol{\psi}_{2}(r ; \vec{k})=\left(-g g^{r r}\right)^{-\frac{1}{4}}\left(\begin{array}{c}0 \\ 0 \\ \boldsymbol{\Phi}_{2}\end{array}\right) \times \sqrt{\frac{r_{-}}{R^{2}}} \times\left(\frac{r_{-}}{R^{2}}\right)^{\nu_{k}}$

with 


$$
\begin{aligned}
& \boldsymbol{\Phi}_{1}=\left(\begin{array}{c}
v_{1} \\
v_{2}
\end{array}\right) \equiv \frac{1}{W} a_{+}\left(k_{0}, k\right) \Psi_{-}^{(0)} \\
& \boldsymbol{\Phi}_{2}=\left(\begin{array}{c}
\tilde{v}_{1} \\
\tilde{v}_{2}
\end{array}\right) \equiv \Phi_{1}(\vec{k} \mapsto-\vec{k})
\end{aligned}
$$

and $W=-i v_{+}^{T} \sigma^{2} v_{-}, a_{+}\left(k_{0}, k\right)=\tilde{c}_{1}\left(k-k_{F}\right)+\tilde{c}_{2} k_{0}+\cdots$, where $\tilde{c}_{1,2} \sim \frac{R^{2}}{r_{-}}$. The explicit spinors are

$$
\begin{aligned}
\Psi_{ \pm}^{(0)} & \approx v_{\mp}\left(\frac{r-r_{-}}{R_{2}^{2}}\right)^{ \pm \nu_{k}} \\
v_{ \pm} & =\left(\begin{array}{c}
m R_{2} \pm \nu_{k} \\
\frac{k R}{r_{-}} R_{2}+e_{R} \sqrt{\frac{\tilde{\alpha}}{3}}
\end{array}\right),
\end{aligned}
$$

with $R_{2}=R / 2 \sqrt{3}$, and

$$
\nu_{k}=\sqrt{m_{k}^{2} R_{2}^{2}-\frac{\tilde{\alpha}}{3} e_{R}^{2}}, \quad m_{k}^{2} \equiv m^{2}+\frac{k^{2} R^{2}}{r_{-}^{2}} .
$$

Note that for pure $\mathrm{AdS}_{2}$, the soft wave-functions simplify

$$
\boldsymbol{\Phi}_{1}=\Psi_{-}^{(0)} \quad \boldsymbol{\Phi}_{2}=\boldsymbol{\Phi}_{1}(\vec{k} \mapsto-\vec{k})
$$

Finally, note that the Feynman propagator for the soft part in (3.19)-(3.20) is given by

$D_{F}\left(r, r^{\prime} ; k\right)=\boldsymbol{\psi}_{\alpha}(r, k)\left(\int \frac{d \omega}{2 \pi} \frac{\rho_{B}^{\alpha \gamma}(\omega, \vec{k})}{k^{0}-\omega}\right) \overline{\boldsymbol{\psi}_{\gamma}}\left(r^{\prime}, k\right)$

with the boundary spectral function $\rho_{B}(\omega, \vec{k})$, and the normalizable wave function $\psi_{\alpha}(r, \vec{k})$ for the Dirac equation in curved $\mathrm{AdS}_{5}$.

\section{APPENDIX C: PARAMETERS $v_{F}, h_{1}, h_{2}, k_{F} / \mu$ ENTERING THE NEAR HORIZON GREEN'S FUNCTION}

We recall that the retarded Green's function (2.13) in the near horizon limit can be recast in the form

$$
\begin{aligned}
\mathcal{G}_{R}^{11}\left(k^{0}, \vec{k}\right) & \approx \frac{\tilde{h}_{1} \mu}{k-\left(\frac{k_{F}}{\mu}\right) \mu-\frac{1}{v_{F}} k^{0}-\Pi\left(k^{0}\right)}\left(\begin{array}{ll}
0 & 0 \\
0 & 1
\end{array}\right) \\
\Pi\left(k^{0}\right) & =\tilde{h}_{2} \mu e^{i \gamma_{k_{F}}}\left(\frac{k^{0}}{\mu}\right)^{2 \nu_{k}}, \\
\nu_{k} & =\sqrt{\tilde{\alpha}}\left(\frac{k^{2}}{\mu^{2}}-\frac{k_{R}^{2}}{\mu^{2}}\right)^{\frac{1}{2}}, \\
\gamma_{k} & =\arg \left(\Gamma\left(-2 \nu_{k}\right)\left(e^{-2 \pi i \nu_{k}}-e^{-\frac{2 \pi}{\sqrt{3}} e_{R} \sqrt{\tilde{\alpha}}}\right)\right),
\end{aligned}
$$

where $k_{R}^{2} / \mu^{2}=\left(e_{R}^{2} \tilde{\alpha}-\frac{1}{4}\left(\tau-\frac{3}{2}\right)^{2}\right) / 3 \tilde{\alpha}$, and $k_{0} / \mu \sim C_{0} / 2 \sqrt{3} \sqrt{\tilde{\alpha}}$ with the dimensionless constant

$$
C_{0}\left(\nu_{k_{F}}\right)=\left(v_{F} \tilde{h}_{2} \operatorname{Im}\left(e^{i \gamma_{k_{F}}}(-1)^{2 \nu_{k_{F}}}\right)\right)^{\frac{1}{1-2 \nu_{k_{F}}}}
$$

The dimensionless parameters $v_{F}, \tilde{h}_{1}, \tilde{h}_{2}$, and $k_{F} / \mu$ that characterize the retarded Green's function (2.13) and (3.20), are in principle determined numerically as in [18] for the 3-dimensional spacetime, and analytically for R-charged black holes as in [19]. Here, we will not carry out the numerical analysis to determine the parameters precisely, but we note that all of them are some functions of $e_{R}, \tau$, and $\tilde{\alpha}$, i.e., $v_{F}\left(e_{R}, \tau, \tilde{\alpha}\right), \tilde{h}_{1}\left(e_{R}, \tau, \tilde{\alpha}\right), \tilde{h}_{2}\left(e_{R}, \tau, \tilde{\alpha}\right)$, and $k_{F} / \mu\left(e_{R}, \tau, \tilde{\alpha}\right)$. Therefore, the dimensionless parameters $v_{F}, \tilde{h}_{1}, \tilde{h}_{2}, k_{F} / \mu$, and the dimensionful parameter $\mu$ are free parameters. The parameter $\tilde{h}_{1}$ drops out in the normalization. The remaining parameter is set to $\tilde{h}_{2}=1$ for simplicity.

\section{APPENDIX D: EFFECTIVE VERTICES}

The soft-to-hard transition vertices entering in the bulk DIS amplitude involve (B5) for the reduction to $\mathrm{AdS}_{2}$ or (B2) in general for the soft part, with the hard part of the wave function given by

$$
\begin{aligned}
& u_{1}=\left(\frac{R^{2}}{r}\right)^{\frac{5}{2}} J_{m R-\frac{1}{2}}\left(\omega_{1} \frac{R^{2}}{r}\right) \\
& u_{2} \equiv 0 .
\end{aligned}
$$

More specifically, for pure $\mathrm{AdS}_{2}$, the transition vertex is simply given by

$$
\Lambda_{11}^{x}\left(z_{2} ; \omega_{1} ; q ; k\right)=C\left(\nu_{k}\right)\left(\frac{r_{-}}{R^{2}}\right)^{\nu_{k}+\frac{1}{2}} q \int_{0}^{\infty} d z_{2} z_{2}^{\frac{3}{2}+\nu_{k}} K_{1}\left(q z_{2}\right) J_{m R-\frac{1}{2}}\left(\omega_{1} z_{2}\right),
$$

with

$$
C\left(\nu_{k}\right)=e_{R} R^{2}\left(m R_{2}+\nu_{k}\right)(2 \sqrt{3})^{-\nu_{k}} .
$$


In general, the transition vertices are of the form

$$
\begin{aligned}
& \Lambda_{11}^{i}\left(r_{2} ; \omega_{1} ; q ; k\right)=-e_{R}\left(\frac{r_{-}}{R^{2}}\right)^{\nu_{k}+\frac{1}{2}} \int d r_{2} \sqrt{-g}\left(-g g^{r r}\right)^{-\frac{1}{4}} \sqrt{g^{i i}} K_{A}\left(r_{2} ; q\right) u_{1}\left(\begin{array}{c}
0 \\
1 \\
0 \\
0
\end{array}\right)^{T} \Gamma\left(\begin{array}{c}
v_{1} \\
v_{2} \\
0 \\
0
\end{array}\right), \\
& \Lambda_{22}^{i}\left(r_{2} ; \omega_{1} ; q ; k\right)=-e_{R}\left(\frac{r_{-}}{R^{2}}\right)^{\nu_{k}+\frac{1}{2}} \int d r_{2} \sqrt{-g}\left(-g g^{r r}\right)^{-\frac{1}{4}} \sqrt{g^{i i}} K_{A}\left(r_{2} ; q\right) u_{2}\left(\begin{array}{c}
0 \\
0 \\
0 \\
1
\end{array}\right)^{T} \Gamma^{i}\left(\begin{array}{c}
0 \\
0 \\
\tilde{v}_{1} \\
\tilde{v}_{2}
\end{array}\right), \\
& \Lambda_{11}^{j}\left(r_{1} ; k ; q ; \omega_{1}\right)=-e_{R}\left(\frac{r_{-}}{R^{2}}\right)^{\nu_{k}+\frac{1}{2}} \int d r_{1} \sqrt{-g}\left(-g g^{r r}\right)^{-\frac{1}{4}} \sqrt{g^{j j}} K_{A}\left(r_{1} ; q\right) u_{1}\left(\begin{array}{c}
v_{1} \\
v_{2} \\
0 \\
0
\end{array}\right)^{T}\left(\begin{array}{c}
\Gamma^{i} \\
1 \\
0 \\
0
\end{array}\right), \\
& \Lambda_{22}^{j}\left(r_{1} ; k ; q ; \omega_{1}\right)=-e_{R}\left(\frac{r_{-}}{R^{2}}\right)^{\nu_{k}+\frac{1}{2}} \int d r_{1} \sqrt{-g}\left(-g g^{r r}\right)^{-\frac{1}{4}} \sqrt{g^{j j}} K_{A}\left(r_{1} ; q\right) u_{2}\left(\begin{array}{c}
0 \\
0 \\
\tilde{v}_{1} \\
\tilde{v}_{2}
\end{array}\right)^{T}\left(\begin{array}{c}
\Gamma \\
0 \\
0 \\
0 \\
1
\end{array}\right) .
\end{aligned}
$$

Using the gamma matrices explicitly, we can simplify the effective vertices (D4). More specifically, we have

$$
\Lambda_{11}^{x}\left(r_{2} ; \omega_{1} ; q ; k\right)=i e_{R}\left(\frac{r_{-}}{R^{2}}\right)^{\nu_{k}+\frac{1}{2}} \int d r_{2} \sqrt{-g}\left(-g g^{r r}\right)^{-\frac{1}{4}} \sqrt{g^{x x}} K_{A}\left(r_{2} ; q\right) u_{1} v_{1},
$$

with the rest of the vertices following by symmetry

$$
\begin{aligned}
& \Lambda_{11}^{x}\left(r_{1} ; k ; q ; \omega_{1}\right)=-\Lambda_{11}^{x}\left(r_{2} ; \omega_{1} ; q ; k\right), \\
& \Lambda_{22}^{x}\left(r_{1} ; k ; q ; \omega_{1}\right)=\Lambda_{22}^{x}\left(r_{2} ; \omega_{1} ; q ; k\right) \equiv 0,
\end{aligned}
$$

and all other components vanishing. Performing the change of variable $r=R^{2} / z$ and setting $z \ll z_{-}$, we can rewrite the integral in (D5) as

$$
\begin{aligned}
\Lambda_{11}^{x}\left(z_{2} ; \omega_{1} ; q ; k\right) & =C\left(\nu_{k}\right) a_{+}\left(k_{0}, k\right) I_{z}\left(\omega_{1} ; q ; k\right) \\
& =C\left(\nu_{k}\right) a_{+}\left(k_{0}, k\right)\left(\frac{r_{-}}{R^{2}}\right)^{\nu_{k}+\frac{1}{2}} \int_{0}^{\infty} d z_{2} z_{2}^{\frac{3}{2}+\nu_{k}} q K_{1}\left(q z_{2}\right) J_{m R-\frac{1}{2}}\left(\omega_{1} z_{2}\right),
\end{aligned}
$$

with

$$
C\left(\nu_{k}\right)=e_{R} R^{2} \frac{\left(m R_{2}+\nu_{k}\right)}{W}(2 \sqrt{3})^{-\nu_{k}}
$$

The integration can be carried out analytically with the result

$$
\begin{aligned}
I_{z}\left(\omega_{1} ; q ; k\right) & =\left(\frac{r_{-}}{R^{2}}\right)^{\nu_{k}+\frac{1}{2}} \int_{0}^{\infty} d z_{2} z_{2}^{\frac{3}{2}+\nu_{k}} q K_{1}\left(q z_{2}\right) J_{m R-\frac{1}{2}}\left(\omega_{1} z_{2}\right) \\
& =\left(\frac{r_{-}}{R^{2}}\right)^{\nu_{k}+\frac{1}{2}} C_{z}\left(\nu_{k}\right) \frac{1}{q^{\left(\nu_{k}+\frac{3}{2}\right)}}\left(\frac{\omega_{1}}{q}\right)^{m R-\frac{1}{2}}{ }_{2} F_{1}\left(\frac{m R+\nu_{k}+3}{2}, \frac{m R+\nu_{k}+1}{2}, m R+\frac{1}{2},-\frac{\omega_{1}^{2}}{q^{2}}\right),
\end{aligned}
$$


with

$$
C_{z}\left(\nu_{k}\right)=2^{\nu_{k}+\frac{1}{2}} \frac{\Gamma\left(\frac{m R+\nu_{k}+3}{2}\right) \Gamma\left(\frac{m R+\nu_{k}+1}{2}\right)}{\Gamma\left(m R+\frac{1}{2}\right)} .
$$

Note that for the special value $\nu_{k}=\nu_{k}^{*}=m R$, the integrand reduces to the one in [21], and can be evaluated exactly as

$$
\begin{aligned}
I_{z}\left(\omega_{1} ; q ; k\right) & =\left(\frac{r_{-}}{R^{2}}\right)^{\nu_{k}+\frac{1}{2}} \int_{0}^{\infty} d z_{2} z_{2}^{\frac{3}{2}+\nu_{k}^{*}} K_{1}\left(q z_{2}\right) J_{m R-\frac{1}{2}}\left(\omega_{1} z_{2}\right) \\
& =\left(\frac{r_{-}}{R^{2}}\right)^{\nu_{k}+\frac{1}{2}} C_{z}\left(\nu_{k}^{*}, q\right) q^{-\left(m R+\frac{5}{2}\right)}\left(\frac{\omega_{1}}{q}\right)^{m R-\frac{1}{2}}\left(1+\frac{\omega_{1}^{2}}{q^{2}}\right)^{-\left(m R+\frac{3}{2}\right)}
\end{aligned}
$$

and $C_{z}\left(\nu_{k}^{*}\right)=2^{m R+\frac{1}{2}} \Gamma\left(m R+\frac{3}{2}\right)$.

\section{APPENDIX E: LOW-X STRUCTURE FUNCTIONS WITH RADIAL DIFFUSION}

Far from the black hole and including diffusion in the radial direction, the structure functions can be written as

$$
\begin{aligned}
& F_{2}\left(x_{A}, q^{2}\right)=\int_{0}^{\infty} \frac{d \omega^{2}}{2} \int \frac{d^{3} k}{(2 \pi)^{3}} \frac{n_{F}(\omega, \vec{k})}{2 E_{k}} F_{2}\left(x_{k}, q^{2}, \omega\right) \\
& F_{1}\left(x_{A}, q^{2}\right)=\int_{0}^{\infty} \frac{d \omega^{2}}{2} \int \frac{d^{3} k}{(2 \pi)^{3}} \frac{n_{F}(\omega, \vec{k})}{2 E_{k}} F_{1}\left(x_{k}, q^{2}, \omega\right),
\end{aligned}
$$

where [40] (see also [41])

$$
\begin{aligned}
F_{2}\left(x_{k}, q^{2}, \omega\right) & =\frac{g_{0}^{2} \rho^{3 / 2}}{32 \pi^{5 / 2}} \int \frac{d z}{z} \frac{d z^{\prime}}{z^{\prime}} P_{A}^{2}\left(z, q^{2}\right) P_{\psi}\left(z^{\prime}, \omega\right)\left(z z^{\prime} q^{2}\right) \frac{e^{\zeta_{k}(1-\rho)}}{\sqrt{\zeta_{k}}} e^{-\frac{\log ^{2}\left(z z z^{\prime}\right)}{\rho \zeta_{k}}} \\
2 x_{k} F_{1}\left(x_{k}, q^{2}, \omega\right) & =\frac{g_{0}^{2} \rho^{3 / 2}}{32 \pi^{5 / 2}} \int \frac{d z}{z} \frac{d z^{\prime}}{z^{\prime}} P_{A}^{1}\left(z, q^{2}\right) P_{\psi}\left(z^{\prime}, \omega\right)\left(z z^{\prime} q^{2}\right) \frac{e^{\zeta_{k}(1-\rho)}}{\sqrt{\zeta_{k}}} e^{-\frac{\log ^{2}\left(z z^{\prime}\right)}{\rho \zeta_{k}}},
\end{aligned}
$$

with

$$
\begin{aligned}
& P_{A}^{2}\left(z, q^{2}\right)=(q z)^{2}\left(K_{1}^{2}(q z)+K_{0}^{2}(q z)\right) \\
& P_{A}^{1}\left(z, q^{2}\right)=(q z)^{2} K_{1}^{2}(q z) \\
& P_{\psi}\left(z^{\prime}, \omega\right)=z^{\prime-3} \times z^{\prime 5} J_{m R-1 / 2}^{2}\left(\omega z^{\prime}\right)
\end{aligned}
$$

$\rho \equiv 2 / \sqrt{\lambda}, \quad \zeta\left(z, z^{\prime}, \lambda, q^{2}, x_{k}\right) \equiv \log \left(\frac{z z^{\prime}}{\sqrt{\lambda}} \frac{q^{2}}{x_{k}}\right), \quad$ and $\quad g_{0}^{2} \equiv \frac{\kappa_{5}^{2}}{R^{3}}=$ $4 \pi^{2} / N_{c}^{2}$.

\section{APPENDIX F: BLACK HOLE WITH $e_{R}^{2} \tilde{\alpha}<\frac{1}{4}(m R)^{2}$ OR $k_{R}^{2}<0$}

Near the black hole the bulk fermions get modified in the infrared as illustrated in Fig. 2. The modification depends quantitatively on their charge and mass near the horizon as we discussed earlier. For fermions with $e_{R}^{2} \tilde{\alpha}<\frac{1}{4}(m R)^{2}$ or $k_{R}^{2}<0$, the modification is universal and follows from the reduced $\mathrm{AdS}_{2} \times \mathrm{R}^{3}$ geometry. More specifically, for hard R-probes with large $q^{0}$ in the DIS kinematics, only $\mathcal{G}_{R}\left(k^{0}, \vec{k}\right)$ is modified close to the horizon, since $\mathcal{G}_{R}\left(\omega_{1}, k+q\right)$ carries a large momentum and is mostly unmodified in the ultraviolet. In this regime, the holographic fermions form a disc of radius $k_{R}$ in momentum space as we noted earlier, with large real and imaginary parts. With this in mind, we have

$$
\operatorname{Im} \mathcal{G}_{R}^{11}\left(\omega_{1}, k+q\right) \operatorname{Im} \mathcal{G}_{R}^{11}\left(k^{0}, \vec{k}\right) \rightarrow \operatorname{Tr}\left(\left(\sigma_{1}\left(k^{0}+q^{0}\right)-i \sigma_{2}\left(k_{x}+q_{x}\right)-\omega_{1}\right) \pi \delta\left((k+q)^{2}+\omega_{1}^{2}\right) \times \operatorname{Im} \mathcal{G}_{R}^{11}\left(k^{0}, \vec{k}\right)\right)
$$

Here $\mathcal{G}_{R}^{11}=\mathcal{G}_{R} \operatorname{diag}(0,1)$ is given in (2.10) for small $\omega$. Again note the emerging non-Fermi liquid scaling for $\nu_{k}<\frac{1}{2}$ with the transition to a normal Fermi liquid for $\omega \approx \omega_{c}$ as discussed earlier. Using the vertex for pure $\mathrm{AdS}_{2}(\mathrm{D} 2)$, we can rewrite (3.26) as 


$$
\operatorname{Im} \tilde{G}_{x x}^{F}(q)=q^{2} C_{\theta}(-1) \int_{0}^{\infty} \frac{d \omega_{1}^{2}}{2} \int d k k^{2} C^{2}\left(\nu_{k}\right) I_{z}^{2}\left(\omega_{1} ; q ; k\right) \operatorname{Re} I_{k^{0}}\left(\omega_{1}, q, x_{A}\right)
$$

with $C_{\theta}=1 /\left(12 \pi^{2}\right)$, and the real part of $I_{k^{0}}$ is

$$
\begin{aligned}
\operatorname{ReI}_{k^{0}}\left(\omega_{1}, q, x_{A}\right) & =\operatorname{Re} \int_{-\infty}^{\infty} \frac{d k^{0}}{2 \pi} \operatorname{Tr}\left(\mathcal{G}_{F}^{11}\left(\omega_{1}, k+q\right) \mathcal{G}_{F}^{11}\left(k^{0}, \vec{k}\right)\right) \\
& =\operatorname{Re} \int_{-\left|q^{0}\right|}^{0} \frac{d k^{0}}{2 \pi} \operatorname{Tr}\left(\left(\sigma_{1}\left(k^{0}+q^{0}\right)-i \sigma_{2}\left(k_{x}+q_{x}\right)-\omega_{1}\right) \pi \delta\left((k+q)^{2}+\omega_{1}^{2}\right) \operatorname{Im} \mathcal{G}_{R}^{11}\left(k^{0}, \vec{k}\right)\right) \\
& \approx \int_{-\omega_{c}}^{0} \frac{d k^{0}}{2 \pi}(-1) \omega_{1} \pi \delta\left((k+q)^{2}+\omega_{1}^{2}\right) \operatorname{Im} C(\vec{k})\left(k^{0}\right)^{2 \nu_{k}}
\end{aligned}
$$

By first doing the integral over $\omega_{1}$ in (F2) and using the delta function in (F3), we finally obtain

$$
\begin{aligned}
\operatorname{Im} \tilde{G}_{x x}^{F}(q) \approx & \frac{C_{\theta}}{4} \int d k k^{2} C^{2}\left(\nu_{k}\right) q^{2} I_{z}^{2}\left(\sqrt{s_{k}} ; q ; k\right) \sqrt{s_{k}} \operatorname{Im}\left(\frac{-C(\vec{k})\left(-\omega_{c}\right)^{2 \nu_{k}+1}}{2 \nu_{k}+1}\right), \\
\approx & \frac{C_{\theta}}{4}\left(\frac{1}{q^{2}}\right)^{\nu_{k}+\frac{1}{2}} \int d k k^{2} C^{2}\left(\nu_{k}\right) C_{z}^{2}\left(\nu_{k}\right) x_{k}^{\nu_{k}+5 / 2}\left(1-x_{k}\right)^{m R-1 / 2}{ }_{2} F_{1}^{2}\left(\frac{m R+\nu_{k}+2}{2}, \frac{m R-\nu_{k}+1}{2}, m R+\frac{1}{2}, 1-x_{k}\right) \\
& \times \sqrt{s_{k}} \operatorname{Im}\left(\frac{-C(\vec{k})\left(-\omega_{c}\right)^{2 \nu_{k}+1}}{2 \nu_{k}+1}\right),
\end{aligned}
$$

with $k^{0}$ fixed to $\omega_{c}$ in $s_{k}=-(k+q)^{2}$. We have defined $x_{k}=-q^{2} / 2 k \cdot q, x_{A}=q^{2} / 2 E_{A} q_{x}$, and made use of the DIS kinematics to approximate $s_{k} \approx-q^{2}\left(1-1 / x_{k}\right),\left|q^{0}\right| \approx q_{x}$.

[1] M. R. Adams et al. (E665 Collaboration), Phys. Rev. Lett. 68, 3266 (1992); M. R. Adams et al. (Fermilab E665 Collaboration), Phys. Lett. B 287, 375 (1992); M. R. Adams et al. (E665 Collaboration), Z. Phys. C 67, 403 (1995).

[2] P. Amaudruz et al. (New Muon Collaboration), Nucl. Phys. B441, 3 (1995).

[3] J. Gomez et al., Phys. Rev. D 49, 4348 (1994).

[4] A. C. Benvenuti et al. (BCDMS Collaboration), Phys. Lett. B 189, 483 (1987).

[5] I. Balitsky, Nucl. Phys. B463, 99 (1996); Y. V. Kovchegov, Phys. Rev. D 60, 034008 (1999).

[6] K. J. Golec-Biernat and M. Wusthoff, Phys. Rev. D 59, 014017 (1998).

[7] C. Adloff et al. (H1 Collaboration), Eur. Phys. J. C 13, 609 (2000); Phys. Lett. B 520, 183 (2001); Eur. Phys. J. C 21, 33 (2001).

[8] S. Chekanov et al. (ZEUS Collaboration), Eur. Phys. J. C 21, 443 (2001).

[9] J. Polchinski and M. J. Strassler, J. High Energy Phys. 05 (2003) 012.

[10] S. J. Brodsky and G. R. Farrar, Phys. Rev. Lett. 31, 1153 (1973).

[11] N. R. F. Braga and A. Vega, Eur. Phys. J. C 72, 2236 (2012).

[12] Y. Hatta, E. Iancu, and A. H. Mueller, J. High Energy Phys. 01 (2008) 063.
[13] K. A. Mamo and I. Zahed, preceding paper, Phys. Rev. D 101, 066013 (2020).

[14] D. T. Son and A. O. Starinets, J. High Energy Phys. 03 (2006) 052; S. S. Lee, Phys. Rev. D 79, 086006 (2009); H. Liu, J. McGreevy, and D. Vegh, Phys. Rev. D 83, 065029 (2011); M. Cubrovic, J. Zaanen, and K. Schalm, Science 325, 439 (2009); S. J. Sin and I. Zahed, J. High Energy Phys. 12 (2009) 015; D. T. Son, Phys. Rev. D 78, 046003 (2008); A. Adams, K. Balasubramanian, and J. McGreevy, J. High Energy Phys. 11 (2008) 059; S. S. Gubser and F. D. Rocha, Phys. Rev. D 81, 046001 (2010); A. Karch, D. T. Son, and A. O. Starinets, Phys. Rev. Lett. 102, 051602 (2009); M. Kulaxizi and A. Parnachev, Nucl. Phys. B815, 125 (2009).

[15] K. A. Mamo and I. Zahed, Phys. Rev. D 100, 046015 (2019).

[16] L. L. Frankfurt and M. I. Strikman, Phys. Rep. 160, 235 (1988); R. P. Bickerstaff and A. W. Thomas, J. Phys. G 15, 1523 (1989); M. Arneodo, Phys. Rep. 240, 301 (1994).

[17] A. Chamblin, R. Emparan, C. V. Johnson, and R. C. Myers, Phys. Rev. D 60, 064018 (1999).

[18] T. Faulkner, H. Liu, J. McGreevy, and D. Vegh, Phys. Rev. D 83, 125002 (2011).

[19] S. S. Gubser and J. Ren, Phys. Rev. D 86, 046004 (2012).

[20] J. H. Gao and B. W. Xiao, Phys. Rev. D 81, 035008 (2010). 
[21] J. Polchinski and M. J. Strassler, J. High Energy Phys. 05 (2003) 012.

[22] M. Rho, S. J. Sin, and I. Zahed, Phys. Lett. B 466, 199 (1999); R. A. Janik and R. B. Peschanski, Nucl. Phys. B586, 163 (2000); E. Shuryak and I. Zahed, Ann. Phys. (Amsterdam) 396, 1 (2018).

[23] R. C. Brower, J. Polchinski, M. J. Strassler, and C. I. Tan, J. High Energy Phys. 12 (2007) 005; A. Ballon-Bayona, R. C. Quevedo, M. S. Costa, and M. Djuric, Phys. Rev. D 93, 035005 (2016).

[24] M. Edalati, J. I. Jottar, and R. G. Leigh, J. High Energy Phys. 04 (2010) 075.

[25] L. Y. Hung and Y. Shang, Phys. Rev. D 83, 024029 (2011).

[26] D. Jorrin, N. Kovensky, and M. Schvellinger, J. High Energy Phys. 04 (2016) 113.

[27] E. J. Moniz, I. Sick, R. R. Whitney, J. R. Ficenec, R. D. Kephart, and W. P. Trower, Phys. Rev. Lett. 26, 445 (1971).

[28] E. J. Moniz, Phys. Rev. 184, 1154 (1969).

[29] P. Amaudruz et al. (New Muon Collaboration), Nucl. Phys. B441, 3 (1995).

[30] M. Arneodo et al. (New Muon Collaboration), Nucl. Phys. B481, 23 (1996).
[31] J. Gomez et al., Phys. Rev. D 49, 4348 (1994).

[32] S. Malace, D. Gaskell, D. W. Higinbotham, and I. Cloet, Int. J. Mod. Phys. E 23, 1430013 (2014).

[33] A. Bodek et al., Phys. Rev. Lett. 50, 1431 (1983).

[34] A. C. Benvenuti et al. (BCDMS Collaboration), Phys. Lett. B 189, 483 (1987).

[35] J. Seely et al., Phys. Rev. Lett. 103, 202301 (2009).

[36] M. Arneodo et al. (New Muon Collaboration), Nucl. Phys. B441, 12 (1995).

[37] M. Arneodo et al. (European Muon Collaboration), Nucl. Phys. B333, 1 (1990).

[38] K. Ackerstaff et al. (HERMES Collaboration), Phys. Lett. B 475, 386 (2000); 567, 339(E) (2003).

[39] N. Iqbal, H. Liu, and M. Mezei, Lectures on Holographic Non-Fermi Liquids and Quantum Phase Transitions, String Theory and Its Applications (World Scientific, Singapore, 2012), pp. 707-815; T. Faulkner, N. Iqbal, H. Liu, J. McGreevy, and D. Vegh, Phys. Rev. D 88, 045016 (2013).

[40] R. C. Brower, M. Djuric, I. Sarcevic, and C. I. Tan, J. High Energy Phys. 11 (2010) 051.

[41] N. Kovensky, G. Michalski, and M. Schvellinger, J. High Energy Phys. 10 (2018) 084. 